\title{
Managing Resilience in Carrier Grade Networks: Survey, Open Issues and Trends
}

\author{
Wilson Ramirez, Xavier Masip-Bruin, Eva Marín-Tordera, Sergio Sanchez-Lopez \\ Advanced Network Architectures Lab (CRAAX), Technical University of Catalonia (UPC), Spain \\ Emails: \{wramirez, xmasip, eva, sergio\}@ac.upc.edu
}

\begin{abstract}
IP/MPLS and Optical technologies are the foundations of current Carrier-Grade Networks (CGNs), due to both the flexibility of IP/MPLS to provide services with distinct requirements and the high transport capacity offered by new Optical technologies, such as Elastic Optical Networks (EONs). However, despite of the widespread adoption of these two technologies, interoperability issues still impact on key network features, in particular on resilience capabilities. Resilience is gaining momentum in recent years due to the advent of new CGN scenarios, such as Data Center Networks (DCNs), where a link or a node failure might lead to a substantial traffic loss. We consider that any potential contribution on the resilience arena, must be built on top of a solid knowledge of available proposals emphasizing most appealing research trends and the limitations affecting the management of resilience in CGNs. Aligned to this scenario, the main goals of this article are: (1) compile distinct approaches for managing resilience; (2) describe in a comprehensive manner, the challenges faced by CGN operators in order to manage resilience; (3) show why current solutions for managing resilience do not completely address the interoperability issues present in multilayer CGNs with multi-vendor settings, and; (4) provide insights for future trends.
\end{abstract}

Index Terms-Multi-layer networks, Resilience, Carrier-Grade Networks.

\section{INTRODUCTION}

For many years, Carrier-Grade Networks (CGN) have undergone substantial changes, mainly driven by the everincreasing demand for transmission capacity, recently pushed by the set of emerging -and those unforeseen- as well services offered to users, supported by the fast development of novel technologies. At present, CGNs are based on a multi-layer network model where IP and Multiprotocol Label Switching (MPLS) technologies are used as the service layer, whereas optical technologies, such as Wavelength Division Multiplexing (WDM) are used as the transport medium [1]. While it is widely accepted that this layered structure brings key benefits to the overall network performance it is also recognized that strict demands about layers coordination are required.

Unfortunately, from a management perspective, both network layers (IP/MPLS and Optical) are highly isolated from each other. The isolation between these two network layers causes an unnecessary duplication of functions and network personnel, which substantially impacts on both the network carriers' business models and the overall network performance. Despite tones of research efforts have been devoted to the control plane arena [2], [3], scarce technological advances addressing the management plane for IP/MPLS and Optical network layers (i.e., multi-layer), have been issued in the last years. As a consequence, the required layers interworking framework, setting the suite of automated coordination actions in multi-layer CGNs has not been substantially accomplished. Thus, additional efforts are still required to propose innovative multi-layer management schemes that might be widely accepted and easily deployed (minimizing operational time and costs) by network manufacturers and providers. Indeed, several network features may be strongly affected by the absence of coordination among network layers (cross-layer coordination), being resilience a critical one.

Resilience is the capability of a network to endow link/node failures. A network with resilience capabilities assures the sustainability of the provided services. Indeed, this a feature highly demanded by CGN operators, since a link failure might lead to a large amount of traffic loss. Therefore, CGNs must endow failures in an agile and efficient manner, i.e, recover the affected traffic as faster as possible and using the minimum amount of network resources to do so.

Notice that the set of required interactions between the IP/MPLS and the Optical network layers to execute the actions for providing resilience, such as the setup of a new lightpath cannot be accomplished in current multi-layer CGNs, without some degree of human intervention. Moreover, the lack of layers coordination is not only motivated by a slow and poor technology progress. Unluckily, control plane operations of IP/MPLS and Optical network layers are also limited by the interoperability issues found in multi-vendor environments. As a matter of fact, for a Network Management System (NMS), the management of Network Elements (NEs) from different vendors and technologies could be a difficult task, mainly fueled by the absence of a vendor-agnostic configuration data model.

The advent of control plane proposals, such as Automatically Switched Optical Network (ASON), Generalized MultiProtocol Label Switching (GMPLS), and Software-Defined Networking (SDN), have substantially addressed several of the interoperability issues related to control plane operations [4], [5], [6]. However, even though recent proposals such as NETCONF [7] have paved the way to cope with the interoperability challenges on the management plane, three major issues yet hinder the advances in this area: 1) the lack of Coordination, which refers to the low level of interaction between and within different networks layers; 2) the Correlation-Problem, which affects the collection of Network State Information (NSI) among network layers, and; 3) the Integration with third-party systems, which includes the issues affecting the interaction be- 
tween IP/MPLS and Optical network layers with new network paradigms, such as the Path Computation Element (PCE) or OpenFlow controllers. We consider interoperability challenges as the main reason preventing resilience management in multilayer CGNs to be fully accomplished.

The main contribution of this article is to delve into the set of challenges faced by operators to manage resilience in multi-layer CGNs. To this end, we provide a constructive criticism elaborating on why the absence of interoperability between the management plane of both IP and Optical network layers significantly diminishes the coordination of recovery actions, and why, in practice, the management of resilience in multi-layer CGNs has not been fully accomplished at present. In addition, we provide insights of future trends concerning multi-layer resilience, as well as some directions to reveal the resilience solution with greater acceptance among network operators.

Similar studies can be found in [8], [9], where authors describe the operation of several resilience strategies for multilayer CGNs. However, this paper is not limited to describe the underlying mechanism of multi-layer resilience strategies, but rather we also focus on the open issues that must be solved (from a CGN operator perspective) related to both control and management planes, to achieve an efficient management of resilience. Moreover, we provide the most recent trends related to the management of resilience in CGNs. A more similar study can be found in [10]. However, this study solely focuses on control plane requirements

The rest of this article is organized as follows. Section II presents in a nutshell the evolution of Transport and Network technologies, clearly introducing the issues to be addressed in order to properly manage resilience in multi-layer CGNs. Section III compiles in a comprehensive manner a survey of resilience schemes as well as the most appealing future trends for managing resilience in multi-layer CGNs. Section IV delves into the current challenges affecting the management of resilience. This section also distils the negative effects of the lack of interoperability between the management planes of both IP/MPLS and Optical technologies on the management of resilience. Section V provides trends and directions related to the management of resilience in CGNs. Finally, section VI presents the general conclusions.

\section{SHIFTING THE PARADIGM FROM TRANSPORT NETWORKS TO RESILIENT MULTI-LAYER CGNS}

This section provides a quick overview on how transport (used for the transport medium) and network (used to provide a service) technologies have evolved in the last decades. We show that substantial modifications have been made over transport and network technologies mainly driven by the bandwidth requirements of new applications and services such as, real time gaming, cloud computing, network resources virtualization, among others. Finally, this section briefly introduces how despite the constant evolution of both transport and network technologies, the proper management of resilience in multilayer CGNs is not totally addressed in practice.

\section{A. Evolution of Transport and Network Technologies}

Fig. 1 depicts the evolution of transport and network technologies from earliest 80's. Since that time Synchronous Digital Hierarchy (SDH) and Synchronous Optical Network (SONET) have been the standards mostly used as the transport medium of CGNs. SONET has been mostly used in North America, while SDH has been used outside North America. SONET/SDH enables the following features to packet-based technologies, e.g., Frame Relay or Asynchronous Transfer Mode (ATM).

- Interfaces with fiber-optic transmission systems.

- High transmission rates.

- Operational support.

- Low restoration time $(50 \mathrm{~ms})$.

- Grooming of multiple technologies.

Despite these variety of features, SONET/SDH has several issues that hinder its deployment in current CGNs, as follows: (1) Shortest-Path algorithms may not be always used for service provisioning; (2) the protection schemes for SONET/SDH demand the use of dedicated paths (turning into a CAPEX increase), and; (3) provisioning actions such as, set-up and tear-down of lightpaths, are static, i.e., manual (human) intervention is required.

On the other hand, regarding the evolution of the network service layer, during the 80's decade, service providers started to deploy Frame Relay and ATM technologies to offer their services to potential customers. However, these two technologies were eventually replaced by IP/MPLS services in the mid 2000 's, due to several well-known issues, e.g., performance, interoperability and operational costs.

Also, in mid 90's, WDM technologies emerged as an extension for SONET/SDH systems. WDM came up as a promising approach to use the vast amount of fiber bandwidth, leveraging fiber transmission capacity multiplexing. Recent research works claim that the limit of fiber capacity is nearly up to 100 Tbps [11]. Such amount of bandwidth is incredibly higher than the potential bandwidth of any other transmission media. Recent optical transmission testbeds also show that reaching an optical bandwidth capacity above 1 Tbps is not a utopia [12]. Moreover, by means of optical technologies such as WDM, it is possible to achieve alloptical communications, i.e., pure optical data transmission. An all-optical communication model reduces the total powerconsumption, which is highly demanded in CGNs. As a result, WDM technologies are feasible solution for bandwidth hungry services and applications such as, intra-DCN traffic or Video on Demand, among others.

Without overlooking the benefits with regard to the transmission capacity offered by WDM technologies, during the 90's decade, optical nodes had a major limitation related to their ease of configuration. Consequently, great research efforts have been made in optical networking since the early 2000's in order to endow the optical nodes with enough flexibility to be remotely configured. These efforts have led to the creation of Reconfigurable Optical Add Drop Multiplexers (ROADMs). In order to fully exploit the dynamic operation of ROADMs an adequate set of control functionalities is required. This 


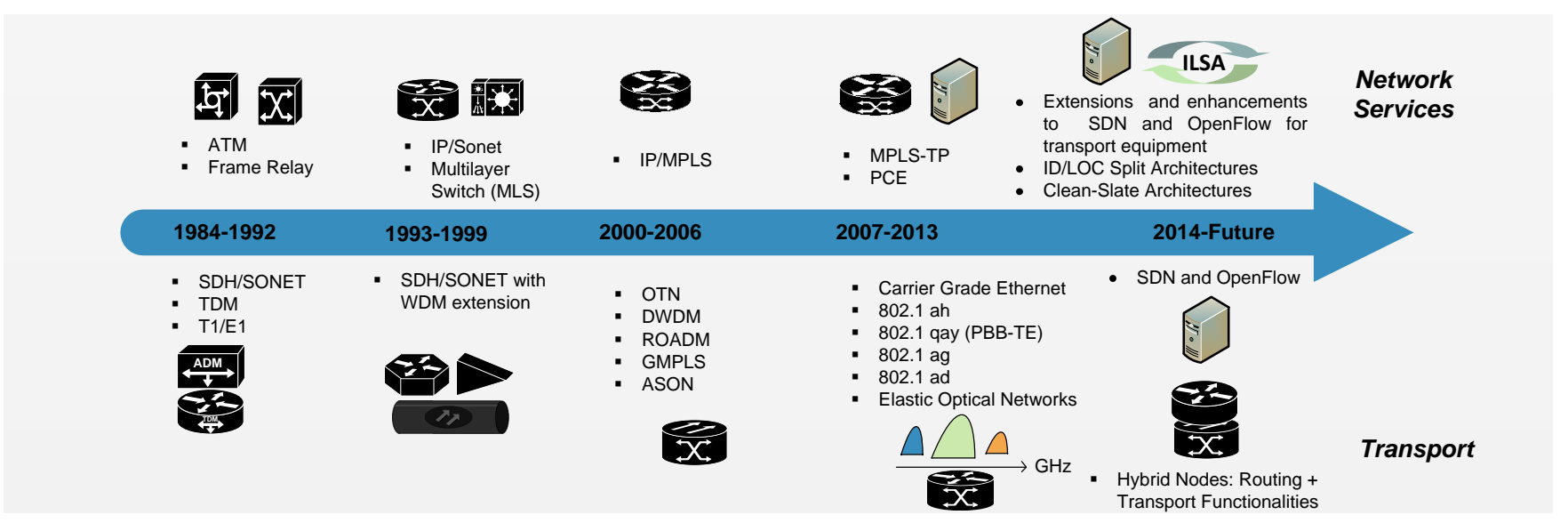

Figure 1. Evolution of Network and Transport Technologies.

control necessity motivated the advent of tentative control plane technologies namely GMPLS and ASON, both aiming to provide optical equipment with the control functions facilitating a variety of novel network features such as, traffic engineering, recovery capabilities, among others.

In mid 2000's, new standards were published to endow transport technologies such as Ethernet, with augmented network features, turning Ethernet into Carrier Grade Ethernet (CGE), as defined by the Metro Ethernet Forum (MEF) [13]. Key building blocks of CGE are the standards IEEE 802.1ad [14], and 802.1ah [15], which increase the level of broadcast segmentation provided by the VLAN stacking employed by the conventional Ethernet. As a result, there was an improvement on the scalability, traffic engineering, and security features. On the other hand, the standards IEEE 802.1ag and the IEEE 802.1qay (PBB-TE) endow CGE with resilience capabilities [16], [17]. Notwithstanding the features provided by CGE, it is worth mentioning that a protocol stack formed by four network layers (IP/MPLS/CGE over Optical transport) might impose an excessive signaling for a resilience scheme. Beyond that, the operational cost inherent to the management of four different technologies is high, actually too high, since each individual network layer is requiring specific operational and maintenance requirements. As a consequence, service providers prefer to deliver their services using IP/MPLS over the Optical layer directly.

In addition, it must be also highlighted the potential duplication and overlapping of functionalities. For example, in a failure scenario all network layers (IP, MPLS, CGE, and Optical) activate their own recovery mechanisms, what could lead to inconsistent NSI (as it is shown in Section III). Despite coordination among network layers is mandatory to guarantee the avoidance of duplicated recovery actions, it still remains as an open issue in current network research.

Another noticeable evolution of the transport layer, was the advent of Elastic Optical Networks (EONs) [18]. EONs provide a flexible-grid spectrum contrary to the conventional $50 \mathrm{GHz}$ fixed-grid spectrum. By means of a flexible-grid spectrum, it is possible to reduce both power and optical resources required for optical transmission. There is not doubt that resilience strategies must be enhanced in order to achieve optimal performance in EON scenarios, because of the new kind of NSI that must be considered for routing, such as, physical link distance and quality of the optical signal. This reasoning is supported by the wealth of studies dealing with the management of resilience in EONs [19], [20], [21].

Also in mid 2000's, IP/MPLS started to become an essential part of the network core of CGNs. This encouraged the network community to provide MPLS with features such as survivability, maintenance tasks, among others. As a result, the MPLS Transport Profile (MPLS-TP) emerged as an initiative of both the Internet Engineering Task Force (IETF) and the Telecommunication Standardization Sector (ITU-T) to expand MPLS for supporting features, commonly demanded by transport technologies [22].

More recent contributions are positioning three different trends: 1) Hybrid Nodes, new network paradigms, and ID/LOC Separation Architectures (ILSA). First, there is a trend in network research consisting in combining the functionalities of optical circuit and packet switching technologies into one single Network Element (NE). This new NE is a hybrid node, commonly referred to as Programmable Hybrid Router (PHR) [23] that can be programmed to enable optical packet switching, circuit switching, low-level electronic packet routing or even all functionalities at the same time. The rationale behind PHRs is to combine the features provided by the IP and Optical NEs, in order to obtain a flexible network architecture, which can be adapted on the fly, to provide a set of heterogeneous services. To this end, hybrid node architectures must support a seamless integration of the management and control planes of both IP and Optical network layers. We consider that this integration facilitates the execution of several network tasks, such as the provisioning of services and the coordination of recovery actions. This novel hybrid architecture is supported by the fact that, network manufacturers already started to introduce this node architecture in their product catalog [24].

Moreover, there is another considering trend emerging network paradigms and technologies, and its potential contribution to network management. In light of this, it is worth mentioning the advent of new network architectures such as Path Computation Element (PCE) and Software Defined Networking (SDN). 
The advent of new network paradigms and third-party systems such as, the PCE and SDN offers new network features, such as advanced path computations, as well as the customized and agile programming of the forwarding plane of IP and Optical NEs. These features are essential for managing resilience in CGNs.

On the other hand, new communication models have recently came up adopting a service-oriented ${ }^{1}$ communication model instead of the conventional host-oriented model. A service-oriented communication model stems on the fact, that users are interested in a particular service independently of who is providing it. Based on this, a connection is attached to an Identifier (ID) representing a set of possible hosts able to provided the demanded service. Conversely to host-oriented communication models where connections are attached to a destination-host location ( IPv4/IPv6 address).

This new network paradigm is motivated by the well-known limitations of the host-oriented model, as well as by the failure of the diverse set of ad-hoc solutions that have been proposed to address these limitations. More specifically, a service-oriented communication model offers an alternative to the traditional "OSIfied IP networks". Two conceptual areas may be considered in network research in order to enable service-oriented communications: 1) Clean-Slate architectures, that is, solutions decoupled from the traditional OSI layered structure (for example adopting information or a service-aware communication model), and; 2) Non-disruptive approaches, i.e., solutions "friendly" to the current layered structure (but still offering service-aware communication capabilities), such as ILSA schemes. Both approaches have become the target for numerous research efforts in the recent years.

Although some contributions may be found in the literature working in both trends [25], [26], CGN operators will surely adopt the one best meeting the pragmatic aspects of their operational networks. In that sense, it is important to notice that network carriers are very reluctant to adopt clean-slate architectures, mainly due to the difficulty of migration tasks, and the potential disruption on their provided services that this migration could promote. Thus, based on this pragmatic feeling, it seems that non-disruptive approaches, such as ILSA schemes, come to be more appealing (at least easy to deploy) than Clean-Slate architectures. The main goal of ILSA proposals is to solve the double functionality problem inherent to the current addressing scheme, and the exhaustion of addresses by assigning an independent set of addresses for identification and location functions.

\section{B. The need for resilience}

The evolution in the network technology cannot be explained without considering the high demanding set of services and applications recently introduced into the market. In addition, and definitely more relevant, it must be also considered the capacities offered to "others" (individual users or service developers) to create new communication scenarios and cooperation frameworks requiring stringent demands on network performance. This network demanding scenario was

\footnotetext{
1 also referred to as information or content oriented communications.
}

fueled some years ago by the introduction of optical technologies that undoubtedly enabled high speed transmission, necessary for the provisioning of services requiring a high amount of bandwidth, e.g., IPTV or Video on Demand (VoD). Current market evolution is envisioning a highly demanding scenario, supported by wide, seamless and high capacity connectivity, all as a whole paving the way to innovative services. These services must be linked to a Service Level Agreement (SLA), responsible for detailing and hence setting the rules and policies to guaranteeing the required network capacities, and demanding resilient end-to-end connectivity. In consequence, the network infrastructure, mainly supported by a multi-layer network consisting in IP/MPLS over Optical networks, must be endowed with resilience mechanisms in order to provide fault tolerance services. Indeed, resilience stands for the capability of a network to maintain the offered services undisrupted in the face of failures.

Resilience can be by achieved by means of Recovery schemes. A proper management of recovery strategies is required to get benefit from the different features inherent to the layers building the multi-layer architecture. Indeed, an appropriate management of this overall setting is evidently required to guarantee and efficient and reliable traffic forwarding. Unfortunately this global interworking between the management and control planes of the IP/MPLS and Optical network layers must face the cross-layer coordination problem, standing for three significant issues, coordination, correlation and integration, nowadays not yet solved. The cross-layer coordination problem in multi-vendor scenarios is one of the interoperability issues that pose major challenges for the management of resilience in multi-layer CGNs. Fortunately substantial modifications have been made over the control plane of both IP/MPLS and Optical technologies with the aim of enabling cross-layer coordination [27]. However, there are still several open issues related to cross-layer coordination. The most relevant one for a CGN operator is the definition of signaling mechanisms required to enable both cross-layer coordination and the exchange of NSI such as, network topology or links statistics in multi-vendor scenarios.

A trend to address this interoperability issue is the one followed by traditional IP vendors such as Cisco or Juniper, which is centered on supporting the integration of Dense (D)WDM transponders in their IP routers. This equipment unification shall reduce the amount of components in the network and the optical transponders cost [28] and also shall facilitate its management, but it increases the interconnection challenges between network vendors at the optical signal level. In other words, an integrated transponder must be compatible with other vendors' transponders, which is not possible at the current state of the art for high rate interfaces [29].

\section{Resilience in Multi-Layer Networks}

This section surveys main contributions addressing resilience in multi-layer networks. Moreover, we introduce to non-expert readers main resilience concepts such as Protection, Restoration and Recovery, which are definitely needed to get a comprehensive understanding of resilience. 


\section{A. Overview of Resilience Concepts}

Resilience capabilities are implemented in terms of recovery mechanisms. The concept of Recovery refers to those actions executed in order to recover the traffic affected by a failure in the network (affecting a link or node). A recovery mechanism can be categorized in two approaches: (1) Protection schemes, and; (2) Restoration schemes.

It is important to remark that the concepts of Protection and Restoration are frequently misunderstood. Even though they refer to similar aspects, both differ on the time defining the recovery actions to be applied. Indeed, recovery actions are applied before or after the failure occurs in protection and restoration schemes respectively.

The protection schemes, applied at the network planning stage, can be mainly categorized in four approaches [30].

1+1 Protection (Proactive Dedicated Protection): This protection strategy employs a dedicated backup path to protect a certain primary path. Traffic is sent along both paths, the primary and the backup, based on a selection criterion, and only the traffic received from one of these paths is chosen at the destination.

1:1 Protection (Standby Dedicated Protection): Under this type of protection scheme the affected primary path's traffic is solely sent along a backup path when the primary path fails. Under normal conditions, a backup path can be used to convey traffic from another primary path, in order to for example, avoid network congestion.

1:N Protection (Shared Protection): With this protection strategy several protection paths $(\mathrm{N})$ share common network resources (optical wavelengths, IP bandwidth) along overlapping links. When a primary path fails the affected traffic is sent along the backup path, and in that case the remaining $\mathrm{N}-1$ primary paths will be unprotected.

M:N Protection $(\mathbf{M} \leq \mathbf{N})$ : Similar to 1: $\mathrm{N}$ protection, but $\mathbf{M}$ paths are used for protecting $\mathrm{N}$ primary paths.

A proactive Dedicated Path Protection (DPP) is usually the scheme employed in practice by network operators, because of its ease of deployment and its low recovery time (50 $\mathrm{ms}$ ). However, due to its proactive nature, a DPP scheme requires a vast amount of network resources to enable path protection $\left(P_{\text {cost }}\right)$. On the contrary, a Shared Path Protection (SPP) scheme has a lower $P_{\text {cost }}$, but the recovery time is higher (over $100 \mathrm{~ms}$ ) in comparison with DPP, mainly because of the time required to switch traffic from the affected primary path to the backup path. In addition, SPP schemes require high signaling overhead. Unfortunately, high signaling overhead adds substantial challenges over both control and management planes.

From the perspective of a CGN designer, it would be optimal to combine the advantages of both DPP and SPP schemes to get low recovery times with low network resources consumption respectively. Driven by this necessity, an innovative protection strategy referred to as Network Coding Protection (NCP) has recently emerged as a promising solution offering protection in an agile and cost-efficient manner. NCP strategies leverage the use of throughput improvement techniques such as Network Coding (NC) jointly with a proactive protection scheme [31].
On the other hand, restoration schemes are reactive in the sense that they employ a recovery action that is dynamically computed upon a failure event. As a consequence, a restoration scheme provides more flexibility for computing a recovery action compared to a protection scheme [32]. In addition, a restoration scheme is suitable for a diverse set of failure scenarios without require over-dimensioning [33], such as multiple simultaneous failure scenarios (caused by catastrophic events such as a hurricane, or an earthquake) [34], [35].

An easy observation reveals that since protection schemes are proactive, they require less signaling to trigger a recovery action compared to a restoration scheme. Therefore, a protection scheme needs less time for recovering traffic, since the recovery action is already computed (usually at the network planning phase). Indeed, the recovery time required by restoration schemes is over $200 \mathrm{~ms}$ [36]. Let $T_{r}$ be the recovery time computed as shown in Equation (1), where $T_{c}$ is the time required to compute and select the protection path and $T_{r s}$ is the time to switch the affected traffic from the primary path to the protection path. For protection schemes, $T_{c}$ is neglected.

$$
T_{r}=T_{c}+T_{r s}
$$

In fact, the signaling required by restoration schemes hinders their deployment, since it may be troublesome in multi-layer CGNs, because a restoration scheme undoubtedly needs cross-layer coordination [32], [37] (see Section IV). In addition, the deployment of a restoration scheme can be troublesome also in multi-vendor network scenarios, because the interoperability of the control and management planes among NEs belonging to different vendors has not been totally achieved (intra-layer coordination). Therefore, both cross-layer coordination and intra-layer coordination are two handicaps for deploying a restoration scheme (later discussed in Section IV).

It must be noticed that both restoration and protection schemes are not mutually exclusive, they can jointly coexist. For instance, in the case of a multiple failure scenario, where several links failed simultaneously (including backup paths) leading to a network state not covered by the deployed protection scheme, a restoration scheme might be needed in order to compute a second backup path for recovering the affected traffic.

Table I summarizes a collection of recovery (protection and restoration) schemes for either IP or Optical networks, i.e., Single-Layer Recovery (SLR) schemes, see Section III.B. For more information regarding SLR schemes the reader can refer to [38].

\section{B. Recovery schemes}

Recovery schemes can be categorized into two main approaches Single-Layer recovery (SLR) schemes and MultiLayer Recovery (MLR) schemes (MLRs). Moreover, MLR schemes are categorized depending on the layer executing the recovery actions required to restore the affected traffic: MultiLayer Recovery Schemes Bottom-UP (MLRBU) and Multi- 
Table I

SINGLE-LAYER RECOVERY SCHEMES FOR IP/MPLS AND OPTICAL NETWORKS.

\begin{tabular}{|c|l|l|}
\hline Network Layer & \multicolumn{1}{|c|}{ Recovery Schemes } \\
\hline IP/MPLS & IP Fast Reroute [40], Redundant Trees [41], NCP [42][31]. & $\begin{array}{l}\text { Concurrent Restoration [43], Shortest-path Tree Restoration } \\
\text { [44], Survivable IP Mapping [45]. }\end{array}$ \\
\hline Optical & $\begin{array}{l}\text { Dedicated Path Switched WDM self-healing ring (DP- } \\
\text { WSHR), Dedicated Path Protection (DPP), Dedicated Path } \\
\end{array}$ & $\begin{array}{l}\text { Local Restoration [45], PCE based restoration [52], Other } \\
\text { related works: [54], [55], [33]. }\end{array}$ \\
& $\begin{array}{l}\text { Protection with Physical Layer Impairments [47], Shared Line } \\
\text { Line Protection (DLP), Redundant Trees [48], Shared sub- } \\
\text { connection Protection [49], p-Cycle Survivability [50], NCP } \\
\text { [51], [31]. }\end{array}$ & \\
\hline
\end{tabular}

Layer Recovery Schemes Top-Down (MLRTD). Fig. 2 shows MLR schemes taxonomy.

SLR schemes do not require cross-layer coordination. Thus, the recovery actions are executed solely within one network layer. Many studies available in the literature argue that SLR schemes are not only inefficient but in some cases ineffective, since in some failure scenarios SLR schemes might not be able to recover the affected traffic [57], [58]. Table I shows a collection of SLR schemes for IP/MPLS and Optical network layers.

Unlike SLR schemes, MLRBU refers to those schemes where upon a failure event, the execution of recovery actions are applied at the bottom network layer, e.g., the Optical layer; in case the affected traffic cannot be restored, then recovery actions are triggered at an upper network layer, e.g., the IP/MPLS layer. On the other hand, the operation of a MLRTD scheme is pretty similar to a MLRBU scheme, though differing on the order network layers trigger recovery actions, thus MLRTD triggers recovery actions from the upper down to the bottom network layer [8]. In this paper we consider that the IP/MPLS layer is on top of the Optical layer, since Optical technologies are commonly used as a transport medium. Other authors refer to the IP/MPLS domain as the client layer and the Optical domain as the service layer.

MLRBU schemes are proposed in [59] and [60], claiming that MLRBU schemes are more agile (low recovery time) compared to MLRTD schemes. Authors in [61] proposed MLRTD schemes, arguing that finer granularity level recovery actions may be obtained in comparison with MLRBU. This is an advantage in order to select customized restoration paths for traffic flows with distinct characteristics, e.g., data traffic, Video Traffic (sensitive to network delay). This is discussed in detail in Section IV.

It is worth noticing that both MLRBU and MLRTD schemes require cross-layer coordination, in order to sequentially trigger the recovery actions at different network layers. An alternative for scenarios with no cross-layer coordination is referred to as Uncoordinated Multi-Layer Recovery (UMLR). A UMLR scheme triggers recovery actions in a parallel manner at all network layers, what unfortunately can lead to both suboptimal recovery actions (high utilization of network resources used to restore affected traffic) and inconsistent network states, hence limiting its wide adoption.

A MLR scheme can achieve cross-layer coordination by

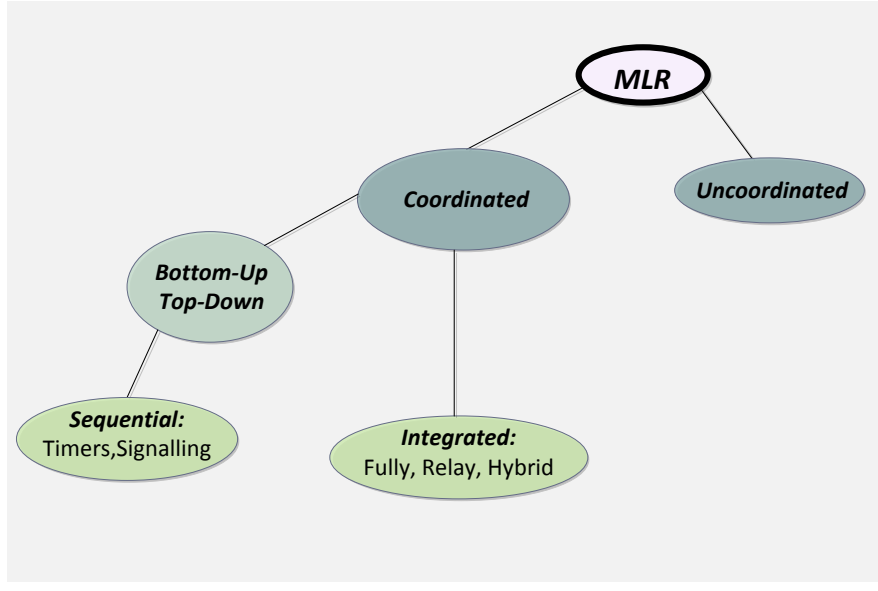

Figure 2. Taxonomy of MLR schemes.

means of two main approaches: 1) Sequential Strategy; and 2) Integrated Strategy.

For a Sequential Strategy a pre-defined mechanism defines when a network layer is unable to restore the affected traffic, hence requiring recovery actions to be triggered at an upper or lower network layer (MLRBU or MLRTD respectively). To this end, two mechanisms are studied in the literature, as follows [8].

- Hold-off timers: mainly based on the use of predefined timeouts. Upon a failure event pops up, the recovery mechanism of each network layer has a predefined timeout defining the time limit to restore the affected traffic. Once this timeout expires, the running recovery mechanism stops, and the recovery mechanism of the upper or bottom network layer is triggered. It is worth mentioning that several timeouts associated to each network layer may be defined (i.e., timeout per failure event), each one customized according to the expected behavior for single failure scenarios.

- Signaling Messages: based on the use of notification messages among the network layers to either stop or start the execution of recovery actions.

On the other hand, an Integrated Strategy scheme is based on collecting NSI for all network layers that is used to select the more appropriate reactive action addressing a failure in the network. A MLR scheme using an integrated strategy can 
follow three approaches.

- Fully Integrated: For this approach, the NEs within the IP/MPLS and Optical network layers play an active role because each of them can coordinate and trigger recovery actions. As it is shown in Fig. 3a, each NE embeds a set of recovery capabilities enabling the control and management of several NEs features. As a result, substantial modifications are needed for conventional routing protocols such as, Open Shortest Path First (OSPF) as well as for GMPLS, ASON, or any other distributed control plane, in order to enable the signaling required to support the advanced routing capabilities needed by an MLR scheme. Authors in [62] propose extensions for conventional routing protocols such as OSPF, to achieve advanced routing capabilities. For more information on this issue the reader is referred to [63].

- Relay MLR: On the other hand, for Relay MLR schemes, all recovery capabilities are embedded into a centralized element referred to as Relay Coordinator, which coordinates all recovery actions in the network. In a Relay MLR scheme, the NEs (IP/MPLS routers and ROADMs from different vendors) play a passive role, because they are restricted solely to send NSI - they do not trigger any recovery action. As it is shown in Fig. 3b, a Relay MLR scheme uses the features of the management plane of both network layers (IP/MPLS and Optical) to coordinate and trigger the required recovery actions. Therefore, a Relay MLR scheme must be able to orchestrate multilayer interactions. This implies the modification of the data models of IP/MPLS and Optical NEs, as well as the communication with Third-party systems such as, a PCE, or SDN. A proposal of a Relay MLR scheme, referred to as ONE Adapter, can be found in [64]. The ONE adapter is a middle box which may communicate with the IP/MPLS and Optical layers and coordinate actions between them. The ONE Adapter enables the dynamic provisioning of a wide and diverse set of services, such as IP service Provisioning, IP offloading actions, and recovery actions.

- Hybrid MLR: As it was already described in Section II, there is a trend in network research referred to as hybrid optical network architectures, which consists in combining the functionalities of optical circuit and packet switching technologies. In these hybrid networks, hybrid nodes also known as PHRs, can be programmed in order to enable packet switching, optical switching, low-level electronic packet routing or even all network features at the same time. In hybrid network scenarios both management and control planes of the Optical and IP/MPLS network layers are merged, as it can be observed in Fig. 3c. Therefore, a Hybrid MLR scheme must be able to orchestrate a variety of services as well as to reconfigure the routing and optical features of the PHRs. To this end, protocols such as ForCES have been proposed [65].

Table. II shows a collection of MLR schemes discussed in this section.
IV. Managing Resilience in MUlti-LAYeR CGNs: The CHALLENGES

As already stated in previous sections, three highly related issues are limiting the deployment of resilience schemes in multi-layer CGNs: (1) Coordination of actions, (2) Correlation of NSI, and (3) Integration with Third-Party Systems. These three issues are the major reasons preventing multi-layer resilience schemes proposed so far, to be totally adopted by network carriers in recent multi-layer CGNs. A comprehensive knowledge of these issues is mandatory to understand why the deployment of MLR schemes is not fully achieved at present. In the following lines, these problems are described in detail.

\section{A. The need for coordination}

The coordination of actions in multi-layer CGNs can be grouped into three sets. (1) The cross-layer coordination (communication among network layers); (2) the intra-layer coordination (communication between NEs belonging to the same network layer technology), and; (3) coordination in hybrid scenarios. In the following lines we exemplify in a compressive manner, the need for both cross and intra-layer coordination in multi-layer CGNs by means of illustrative network scenarios. The posed network scenarios may come up as a result of either a bad network planning (what of course would not be useful for illustrative purposes) or by the negative impact of multiple failures. This section is intended to show that, even when a careful network planning is made, a recovery scheme might not be able to recover the affected traffic in certain network scenarios. Therefore, resilient strategies, i.e., protection and restoration schemes, are required to fully guarantee network resilience against failures.

As described in Section III.B, one of the possible approaches to endow networks with resilience is Single Layer Recovery (SLR) schemes. Unfortunately, SLR schemes are inefficient in multi-layer CGNs. To illustrate the inefficiency of SLR schemes we consider the different network scenarios shown in Fig. 4, modeled by an optical layer (as transport) and an IP/MPLS layer (as service) on top of it. In other words, all IP or IP/MPLS edges are virtual links, i.e., the optical links serve as a transport medium (server links) for the virtual links. In addition, hereinafter, a connection between an optical and a IP node is referred to as cross-layer connection.

Consider that a SLR scheme based on applying recovery mechanisms is applied only at the IP layer. Let's now look at the scenario shown in Fig. 4a, that forwards traffic from router $A$ destined to router $B$ (traffic $A-B$ ) throughout the path $A-D-B$. In the case of a failure affecting router $D$, the SLR deployed at the IP layer can successfully restore the affected traffic, by rerouting the traffic throughout the path $A-C-B$. Hence, in this scenario an SLR recovery strategy will work out. Unfortunately this is not the normal scenario. Let's now consider the scenario shown in Fig. 4b. In this scenario a failure on the optical link $c-d$ pops up, affecting both virtual links $B-D$ and $C-D$, since the optical link $c-d$ is the server link for both virtual links -the traffic on the links $B-D$ and $C-D$ is sent along the optical link $c-d$. In this scenario, the SLR recovery strategy implemented at 


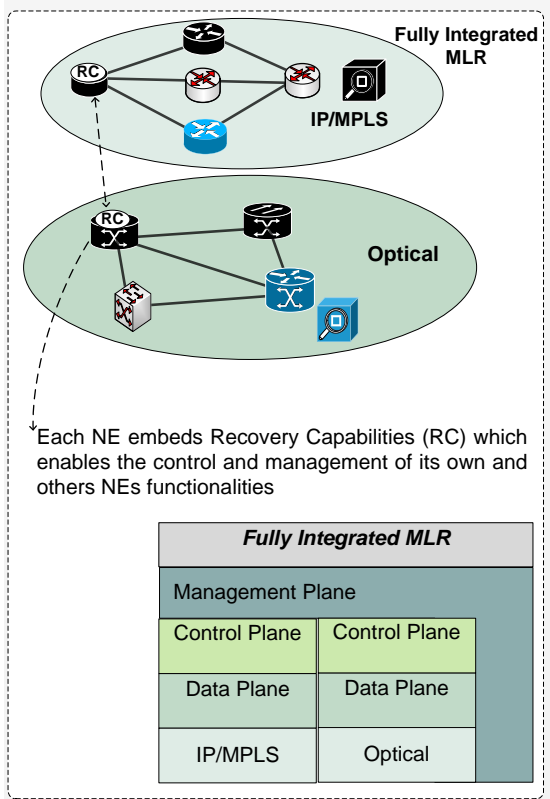

(a)

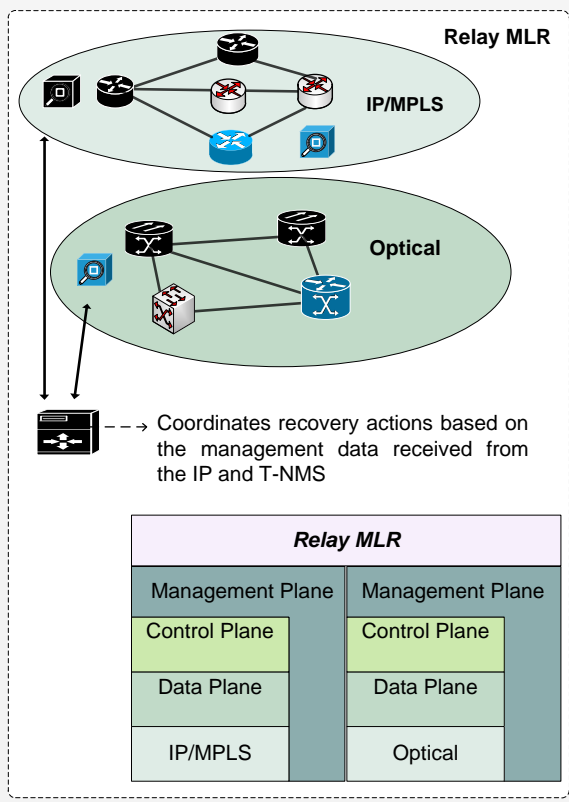

(b)

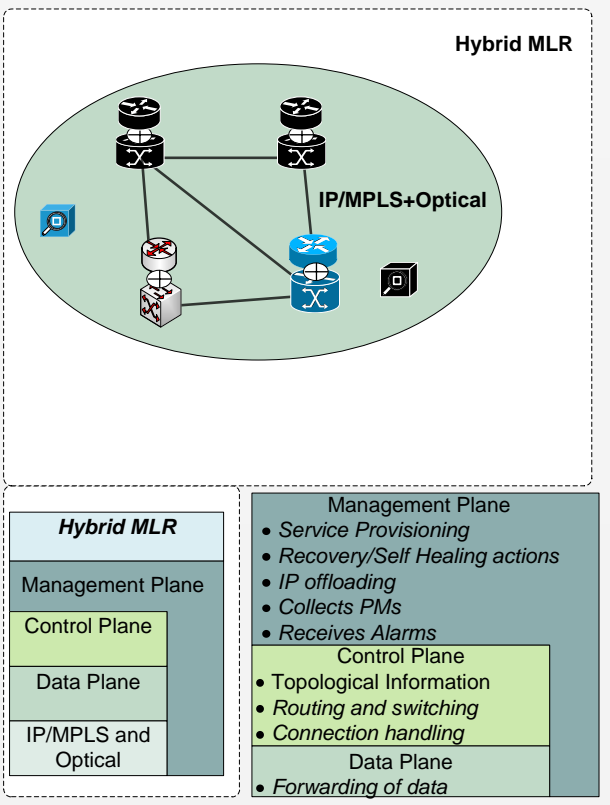

(c)
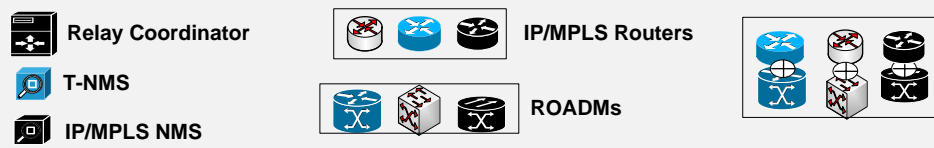

Hybrid Nodes

Figure 3. Integrated Strategies for MLR schemes: a) Fully Integrated MLR; b) Relay MLR; c) Hybrid MLR.

Table II

PROPOSALS FOR MLR SCHEMES.

\begin{tabular}{|c|l|}
\hline MLR schemes & \multicolumn{1}{|c|}{ Proposals } \\
\hline MLRBU & {$[59],[60],[66]$} \\
\hline MLRTD & {$[61]$} \\
\hline Integrated (Fully, Rely and Hybrid) & {$[62],[67],[68],[69],[64],[70],[71],[72]$} \\
\hline
\end{tabular}

the IP layer cannot correctly restore the affected traffic since, nowadays, the IP layer cannot trigger the provisioning of an optical circuit due to cross-layer coordination issues (even though there are recent advances for addressing this issue [70]). Indeed, a possible recovery action could be to set up a new optical circuit between optical nodes $c$ and $d$ along the path $c-a-d$ for reestablishing virtual link $C-D$, and set up a new optical circuit between optical nodes $b$ and $d$ along the path $b-c-a-d$ for reestablishing virtual link $B-D$.

Let's now assume that the recovery strategy is deployed at the optical layer. This scenario is depicted in Fig. 4c and Fig. 4d. The former, Fig. 4c, shows a failure on the optical link c-a, affecting the virtual links $C-A$, and $C-D$ (assuming that the traffic sent along these links is forwarded along the optical link $c-a$ ). This situation may be handled by both: i) setting an optical circuit between optical nodes $c$ and $d$ along the optical path $c-b-d$, to correctly restore the traffic sent on the virtual link $C-D$, and; ii) setting an optical circuit between optical nodes $a$ and $c$ along the optical path $a-b-d-c$, to restore the traffic sent on the virtual link $C-A$. In this case, the SLR strategy would work out.

However, let's now analyze the scenario shown in Fig. 4d, showing what the behavior would be when the failure is at the IP layer. Thus, let's assume a malfunction in router $D$ (e.g., a software failure) affecting its data plane features, such as the traffic sent by router $A$ destined to router $B$ (traf $f i c_{A-B}$ ). This situation may be handled by a recovery action rerouting traf i $_{A-B}$ through router $C$. The issue is that this recovery action can only be deployed at the IP layer, since NEs belonging to the Optical layer are not aware of any failure affecting an IP NE, i.e., the alarms generated by a malfunctioning router will be received only by the NMS of the IP layer (IP-NMS).

Hence, it is clearly observed that SLR strategies may easily fail on their objectives even when a single failure comes up. Obviously, this behavior is even worse in multiple failures scenarios. As a sake of understanding let's consider the network scenario drawn in Fig. 5, showing two simultaneous failure events, a failure on the optical link $a-c$ that affects virtual link $A-C$, and a malfunction in router $D$. A SLR 


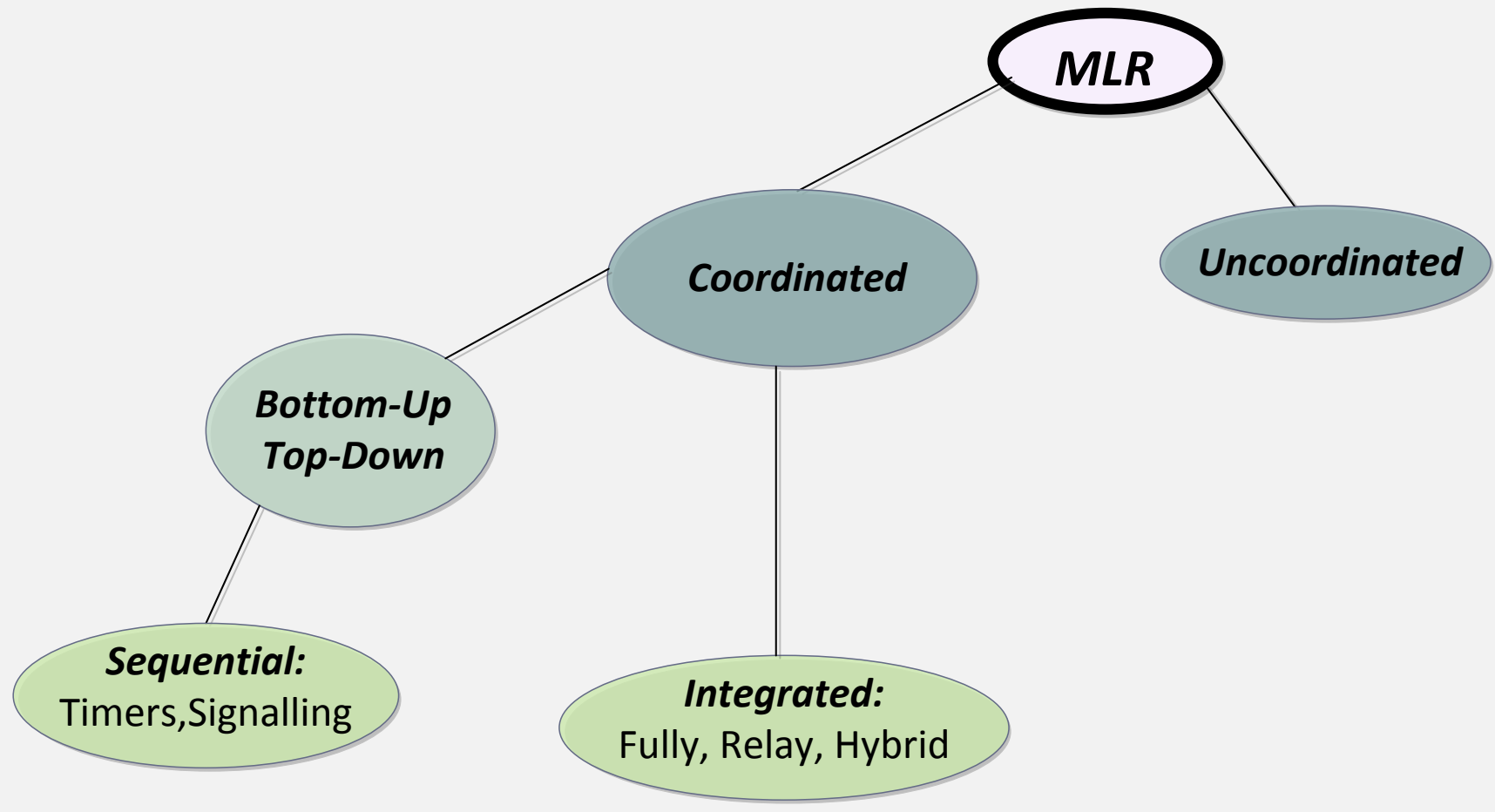

Figure 4. SLR in multi-layer CGNs.

scheme deployed at either the IP or the Optical layer cannot restore the traffic sent by router $A$ destined to router $B$ along the path $A-D-B$, because the Optical layer is not aware of the failure on router $D$, and the IP layer cannot trigger the provisioning of a new circuit between optical nodes $a$ and $c$.

An intuitive reasoning to address the limitations of SLR strategies is to deploy recovery schemes at all network layers in order to cope with all possible failure scenarios. This statement can be exemplified moving back to the network scenario shown in Fig. 5. In this scenario, if the recovery mechanisms on the IP and the Optical layers would coordinate their respective recovery actions, it may be possible both: i) to set up a new optical circuit between optical nodes a and c along the optical path $a-d-c$, to reestablish the virtual link $A-C$, and; ii) to reroute the traffic from $A$ destined to router $B$ along the path $A-C-B$, hence restoring the affected traffic.

Therefore, in any MLR strategy the coordination of recovery actions is highly required; otherwise an uncoordinated MLR scheme may lead to both inconsistent NSI and high $P_{\text {cost }}$. With the aim of illustrating the negative effects of the lack of coordination in multi-layer CGNs, we propose to analyze the network scenario shown in Fig. 6, considering that a MLR strategy is deployed, i.e., recovery strategies at both network layers are reacting to the failure detection. During normal operation the traffic from router $A$ to router $E$ is forwarded

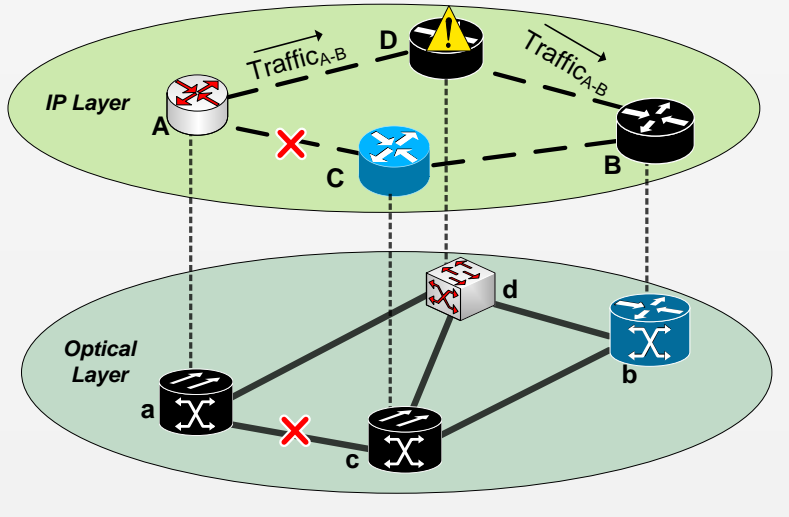

Figure 5. SLR in multi-layer CGNs with multi-failure events.

along the virtual link $A-C$ (along optical link $a-c$ ) using the MPLS label $L 6$. Let's now consider that a failure in the optical link a-c comes up, hence losing the traffic from router $A$ to router $E$. Assuming there is no coordination between the recovery strategies deployed at each layer, two actions are simultaneously triggered, but successfully executed in the following order: i) the recovery mechanism of the IP/MPLS layer reroutes the traffic sent by router $A$ destined to router $E$ by assigning a new MPLS label (for example $L 5$ ) (forwarding this traffic along the IP path $A-D-C-E$ ), and; the recovery 


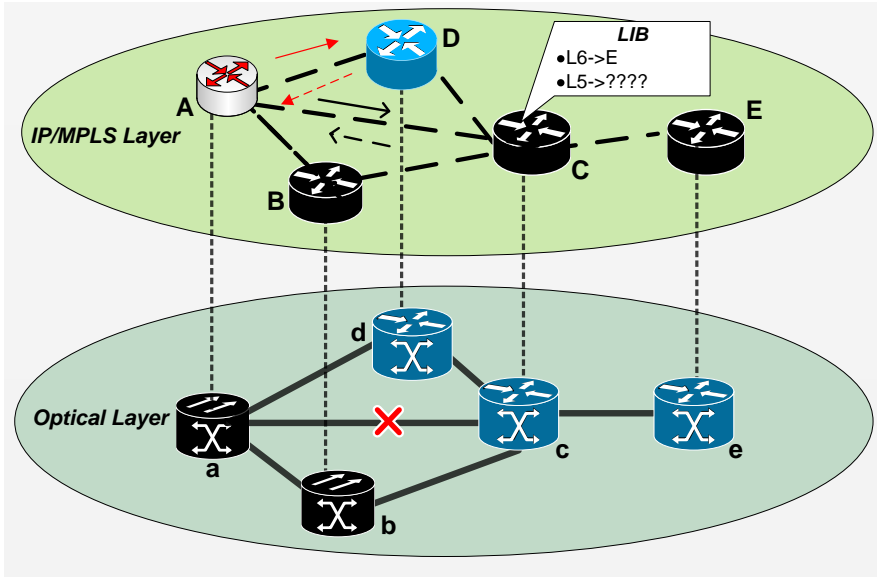

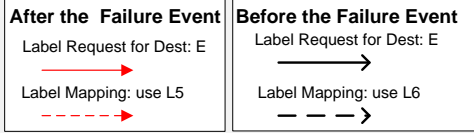

Figure 6. Suboptimal operation of Uncoordinated MLR schemes.

mechanism of the optical layer triggers the provisioning of a new optical circuit between optical nodes $a$ and $c$, along the optical path $a-b-c$, which reestablishes the virtual link $A-C$. Once this virtual link is reestablished, the IP routing mechanism may reroute the affected traffic, without setting a new label at the MPLS layer.

However, notice that a recovery policy on the MPLS layer states that the label $L 5$ (the MPLS label used for all traffic sent from $A$ destined to $E$ ) must be maintained, i.e., not to swap label $L 5$ with another MPLS label such as $L 6$. Therefore, since router $A$ acquired the MPLS label $L 5$ from router $D$, router $C$ does not have label $L 5$ on its Label Information Base (LIB). This will cause router $C$ to drop all traffic with a $L 5$ label.

All the scenarios described so far in this section are valid for SLR and Uncoordinated MLR (UMLR) schemes where the recovery action is computed after the failure event occurs, i.e., restoration schemes. However, the limitations of UMLR and SLR schemes are not restricted to restoration schemes, but also protection strategies need to be considered. The scenario shown in Fig. 7 is posed to demonstrate this behavior. Let's assume that a SLR scheme based on a DPP strategy is deployed at the Optical layer. In addition, notice that within the IP layer broadcast segmentation is enabled by means of OSPF areas [73]. Let's consider a multiple failure scenario, consisting in a failure in both the primary and the backup optical links between optical nodes $a$ and $d$. As a consequence, the virtual link between routers $A$ and $D$ will also fail. In order to restore the affected traffic, it is necessary to set up a new virtual link between routers $A$ and $D$ on the fly, i.e., a restoration scheme is required, since recovery actions included in any protection scheme are defined at the network planning phase.

It is worth mentioning that restoring the affected traffic by rerouting throughout the path $A-B-C-D$ is not possible, since the virtual link $A-B$ belongs to a different OSPF area

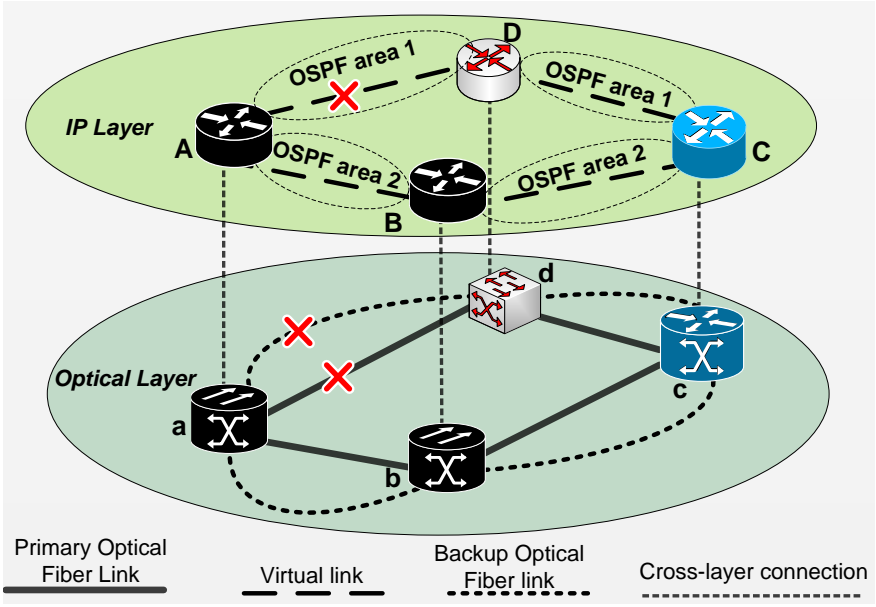

Figure 7. Single layer protection under the presence of multi-failure events.

from link $A-D$.

After the presented analysis, we can correctly assess that if recovery actions are executed only on a single layer basis, there are no guarantees that the affected traffic may be restored for all possible failure scenarios, be either single or multiple. For this reason, a SLR scheme is inefficient in multi-layer CGNs. Moreover, in this subsection we also assess the need for coordination when multiple recovery strategies at different layers are to be applied.

\section{B. Coordination of actions}

In the last section we introduced and illustrated the need for coordination among layers, as well as two sets of potential actions to provide coordination, that is cross-layer coordination and intra-layer coordination. Most significant issues related to both cross-layer and intra-layer coordination are rooted on the fact that each network layer has its own NMS System (IPNMS and T-NMS). Next, we deeply analyze solutions and weaknesses for each coordination set of actions.

1) Intra-layer coordination: As a matter of fact, NEs belonging to the same network technology (intra-layer coordination) are managed through proprietary NMS solutions -each network vendor imposes its own solution. This leads to both an unnecessary duplication of actions and difficult configuration tasks for network administrators, because each vendor enforces its proprietary configuration data model. This situation is magnified at the IP/MPLS layer, since network administrators often use a CLI interface to configure IP/MPLS NEs.

Despite the fact that the use of control plane proposals such as, GMPLS or ASON, is becoming a widespread practice among network operators, neither of these two technologies provides the required granularity level for the provisioning tasks required by recovery actions. This is the rationale driving network operators to rely mainly at the IP/MPLS layer, on the use of management protocols such as Simple Network Management Protocol (SNMP). Nevertheless, SNMP has been mainly restricted to monitoring purposes, hence far from taking over configuration tasks, mainly rooted by two reasons: (i) 
the coarse granularity level offered by SNMP; and, (ii) the lack of a vendor-agnostic data model. These two issues motivated the development of more robust and flexible management protocols such as NETCONF. NETCONF is an XML based protocol that not only provides configuration actions with a high degree of granularity compared to SNMP, but also offers a vendor-agnostic data model termed as YANG [74]. Hence, from a theoretical perspective, NETCONF and YANG can contribute to deal with the intra-layer interoperability issues. However, some challenges remain yet unsolved.

- The development of a common data model designed to embrace the configuration syntax for all different network vendors is a difficult task, because each network vendor adds its own "ingredient" to the protocols running on their NEs. It is also worth mentioning the effects such common data model may have on the current business models, mainly due to the potential reluctance among network vendors about its deployment.

- Despite the management features provided by NETCONF the coordination of recovery actions in multi-layer CGNs deserves further attention.

2) Cross-layer coordination: Two major approaches for multi-layer coordination, as already described in Section III, are sequential and integrated strategies. On one hand, for MLR schemes based on an integrated strategy, two strong limitations exist: i) current control plane protocols do not support the orchestration of complex recovery actions, i.e., actions that involve the simultaneous configuration of several features of a NE, and; ii) The signaling mechanisms needed to achieve cross-layer coordination must be defined, even though there are proprietary solutions already available, there is not a vendor-agnostic signaling mechanisms [75].

On the other hand, for MLR schemes based on a sequential strategy there are several issues related to the escalation mechanisms that must be addressed. For instance, even though the use of Hold-off timers is a pragmatic and easy strategy to implement, computing the optimal value could be troublesome. In fact, while a high Hold-off timer may prolong the restoration time, causing the loss of packets, a low Hold-off timer may not be enough for restoring affected traffic in some network scenarios.

Therefore, replacing Hold-off timers by signaling messages could be another option to manage the sequence of actions. Unluckily, while the use of signaling messages is more efficient than timers [57], it is harder to implement in multivendor environments due to standardization issues. Based on this, the deployment of MLR schemes based on a sequential strategy is limited mainly due to both: i) the required signaling messages for cross-layer communication, and; ii) the layer interoperability issues caused by multi-vendor settings.

3) Coordination in hybrid scenarios: So far in this section, we have analyzed how the lack of coordination impacts on conventional multilayer topologies built by an IP/MPLS layer on top of a WDM network. But, in addition to those conventional multi-layer architectures, we have already introduced in Section III the concept of hybrid node (socalled Programmable Hybrid Router, PHR), as a solution to optimize network management. PHR is based on endowing a NE with the capacity to be programmed to enable optical packet switching, circuit switching, low-level electronic packet routing or even all functionalities at the same time. This theoretically must contribute to support a seamless integration of the management and control planes of both IP/MPLS and Optical network layers. But aligned to the coordination issue, we envision a significant impact on resilience due to the lack of coordination, even for this integrated hybrid architecture. Indeed, several issues must be considered to properly achieve resilience in hybrid networks.

Figure 8 illustrates, as a didactic example, a failure scenario in a hybrid network where node $b$, is a hybrid node programmed as optical switch. Let's assume that router $A$ is sending traffic to routers $B$ and $C$. During normal operation, the traffic from router $A$ destined to routers $B$ and $C$ is groomed at optical node $a$ into optical wavelength $\lambda_{1}$ and then forwarded to hybrid node $b$. Then, hybrid node $b$ drops optical wavelength $\lambda_{1}$ and forwards all traffic to router $B$. Router $B$ receives the traffic destined to itself, and after looking up its routing table, forwards the traffic destined to router $C$ through hybrid node $b$ and optical node $c$ into optical wavelength $\lambda_{2}$.

Let's now assume a failure comes up. In particular, we consider that the cross-layer connection between router $B$ and hybrid node $b$ (marked as 1 in the Fig. 8) fails. Hereinafter, we assume that one cross-layer connection is required per Virtual link. This failure affects the virtual link $A-B$, causing the loss of traffic sent by router $A$ (traffic ${ }_{A-B}$ and traffic ${ }_{A-C}$ ). This is so because the only cross-layer connection available in router $B$ is already used for the virtual link $B-C$. Therefore, there are no cross-layer connections available on router $B$ to establish a new virtual link between router $B$ and router $A$.

A possible recovery action could be to reconfigure the hybrid node $b$ with both IP and optical routing features (this configuration was not assumed before the failure event due to power consumption constraints). Thus, a new virtual link can be established between router $A$ and hybrid node $b$. As a result, the traffic sent by $A$ destined to routers $B$ and $C$ can be forwarded to hybrid node $b$, since this one has IP routing features, i.e., can route IP traffic $A-C$ without being dropped into router $B$. In addition, since hybrid node $b$ also has optical features, it can drop the traffic destined to router $B$. Notice that a virtual link between routers $A$ and $C$ cannot be established because there are no cross-layer connections available in router $C$. Therefore, the strategy to recover from a failure leverages the functionality to be activated on a hybrid node (IP or optical routing), which is a decision that a MLR scheme must take, hence demanding a high level of coordination.

As a conclusion, we can state that the coordination of actions is a major drawback hindering the management of resilience in multi-layer CGNs. Even though the issues related to cross-layer coordination may be minimized by using a simple escalation strategy as hold-off timers, intra-layer coordination can be arduous to achieve in multi-vendor environments. Although major advances have been done on this aspect regarding the control plane, the management plane still demands further development. 


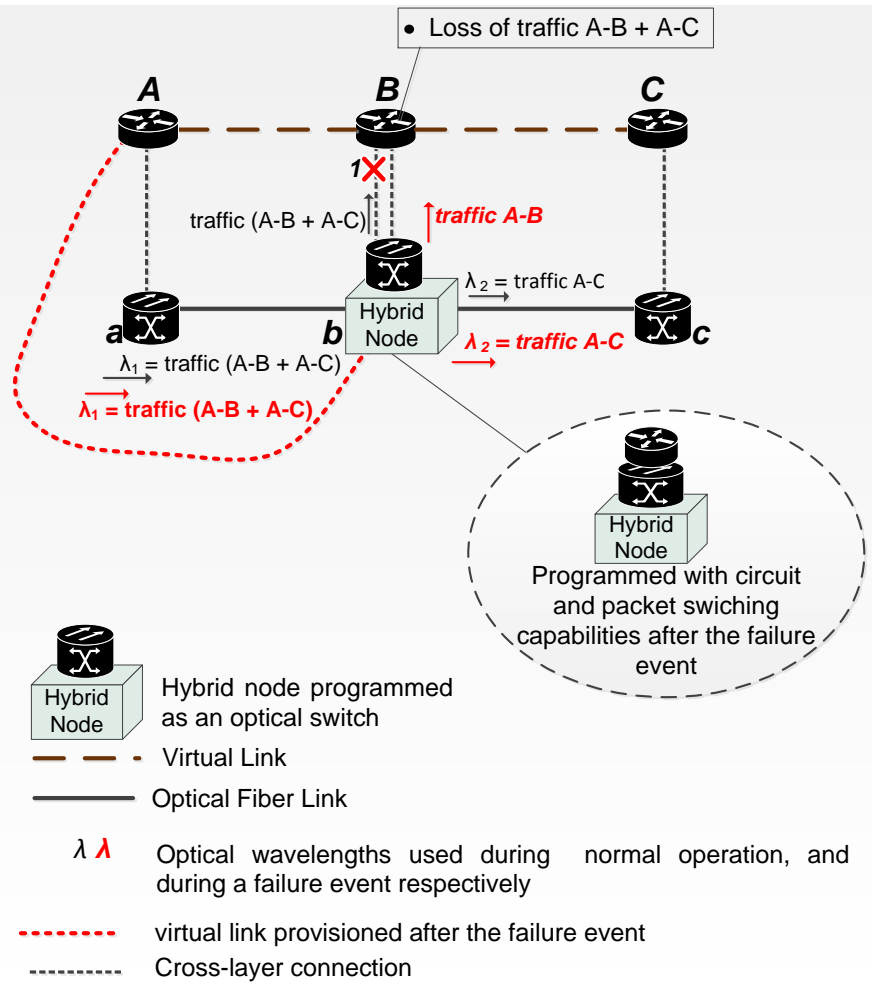

Figure 8. Negative effects of lack of coordination in Hybrid MLR schemes.

\section{Correlation of NSI}

Current multi-layer CGNs do not have the mechanisms required to enable dynamic multi-layer topology discovery in multi-vendor settings. In practice, the multi-layer topology should reflect the set of physical (optical) paths followed by a virtual link, as well as the set of cross-layer connections (connection between an IP/MPLS node and an optical node). In order to build the multi-layer topology, NSI from both IP/MPLS and Optical network layers is required. However, in a real multi-vendor CGN the dynamic discovery of the overall multi-layer network topology could be troublesome, mainly due to both: 1) the IP/MPLS NEs are not aware of the optical network topology and vice-versa, and; 2) the protocols available for topology discovery only operate among NEs belonging to the same vendor.

To the best of our knowledge, the multi-layer topology can only be obtained statically (usually done by observing the status of the virtual links when disconnecting an optical link in the optical layer), since there is not an algorithm to dynamic collect the required multi-layer topological information. In fact, the lack of a multi-layer discovery algorithm strongly limits some of the common operational actions in a multilayer network scenario, such as the alarm correlation and the severity assessment of a failure (see Fig. 9). The complete knowledge of the multi-layer topology is useful for a network operator to know how failures in the optical layer could affect the IP/MPLS virtual links i.e., computation of Shared Risk Link Groups (SRLGs) [76]. Based on this knowledge an MLR scheme is able to estimate the scale of a failure, as well as how the network performance will be affected by potential failures, e.g., assessing the Expected Traffic Loss (ETL) [77].

At present the most realistic solution for alarm correlation in multi-layer networks is the so-called Link Management Protocol (LMP) [78]. Nevertheless, LMP is just a notification protocol; hence, it does not perform advanced computational tasks such as the assessment of the ETL.

The negative effects produced by the lack of NSI correlation are shown in Fig. 9. Let's assume a failure affecting optical link $c-b$. Upper and bottom draws in Fig.9, show the effects of such failure on the overall topology considering the cases that there is no and there is dynamic discovery, collection and correlation of NSI respectively. In the upper scenario there is no NSI correlation, so there is no way to easily estimate what the impact of the failure on the virtual links will be. Opposite to that, in the bottom scenario, the multi-layer topology is known, hence making easier to estimate that the failure affecting link $c-b$ negatively impacts on the traffic sent along virtual links $A-C$ and $C-B$. Knowing such estimation, an MLR scheme might compute the required $P_{\text {cost }}$ with the aim of avoiding possible network congestion. Furthermore, an MLR scheme (specifically, a stateful MLR scheme) could store recovery state information, so in case optical $c-b$ fails again, the recovery paths are foreknown.

In practice, the alarm correlation, the severity assessment of a failure, and the multi-layer topology discovery, are features all adding computational complexity in the NEs, what unquestionably makes its deployment into NEs pretty difficult. The only solution to overcome this computational complexity is to shift to a centralized architecture, where a single centralized-server, such as a Relay MLR scheme, embeds all functionalities.

Indeed, a Relay MLR scheme outperforms a FullyIntegrated scheme in the scenarios where a significant amount of NSI needs to be processed to perform the online computation of a recovery path [79]. As a matter of fact, the implementation of a Fully-Integrated MLR scheme is difficult, because the integration of the control and management planes is an arduous task due to several issues, such as the computational burden and the high complexity added to each NE. This weakness limits correlation-actions such as the multi-layer topology discovery. Notice that the mechanisms provided by OSPF for IP topology discovery may cause both routers congestion, and high convergence time in large network scenarios [80]. This can be more severe if extrapolated to the multi-layer topology scenario. Thus, regarding the execution of correlation-actions, the scalability of Fully-Integrated MLR schemes is very low.

\section{Centralized Network Architectures : A Possible Answer to Resilience Management.}

An integration of third-party systems and new network architectures, such as PCE, SDN, an OpenFlow, may contribute to enhance the resilience level of a MLR scheme. Therefore, as a theoretical principle, any MLR scheme should be able to leverage the features provided by other network architectures in order to improve its performance regarding resilience. In this section we provide some insights on this integration specially for PCE and SDN/OpenFlow scenarios, aiming at 


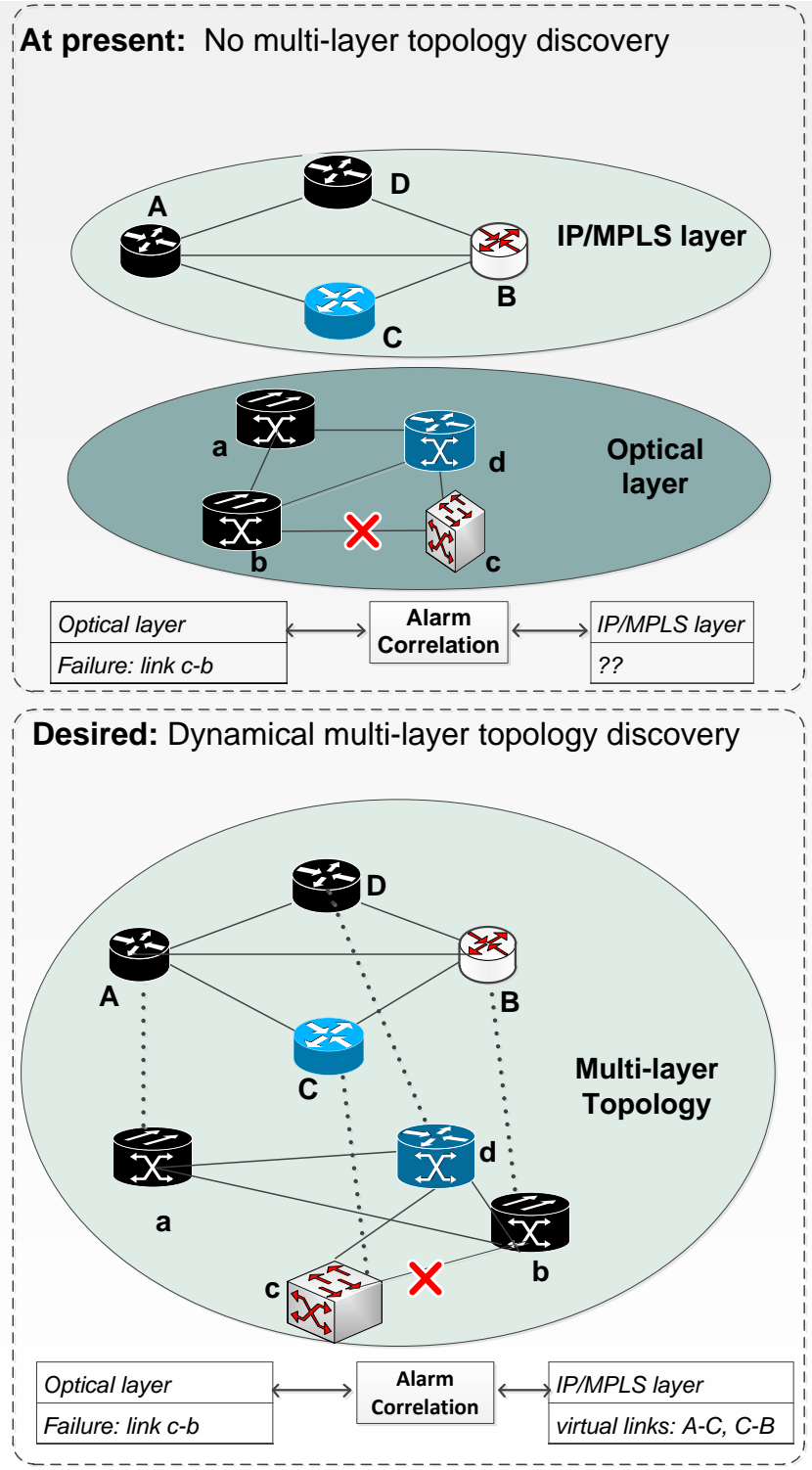

Figure 9. Correlation of NSI in multi-layer in CGNs.

highlighting not only benefits but also existing weaknesses and limitations.

The PCE, as a solution, came up to decouple path computation tasks from data forwarding routers. This is driven by the stringent requirements demanded by new Internet services, which is leading to consider diverse NSI such as, Optical signal conditions, as well as features such as low energy consumption for path computation purposes. Fortunately, the adoption of a centralized routing architecture such as the PCE facilitates an agile (recovery) path computation with low complexity [81]. The advantages provided by PCE schemes fueled significant efforts related to standardize the integration of PCE schemes in current CGNs [82], [83], as well as several scientific studies dealing with PCE utilization in multi-layer CGNs [84], [85].

In order to illustrate the features provided by a PCE scheme we consider the scenario shown in Fig. 10. In this scenario, a

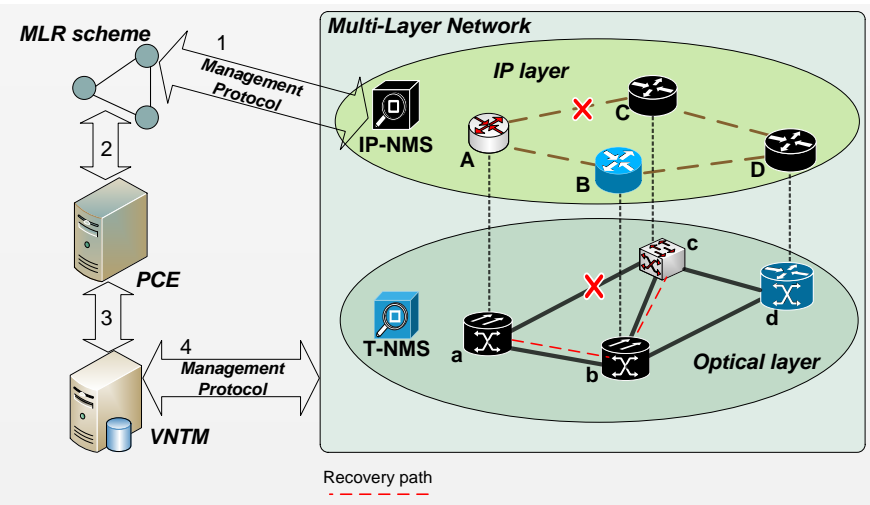

Figure 10. Integration of a PCE in multi-layer CGNs.

PCE interacts with a NE, referred to as the Virtual Network Topology Manager (VNTM) [86]. The VNTM manages the multi-layer topology NSI and it is responsible for provisioning tasks, such as set-up and tear-down of lightpaths.

As it can be seen in Fig. 10, an IP NMS informs a MLR scheme about the failure affecting virtual link $A-C$ (step 1). As a consequence, using the Path Computation Element Protocol (PCEP), an MLR scheme sends a path computation query to a PCE, requesting a new connection between nodes $A$ and $C$, see step 2. Then, the PCE asks the VNTM for the set of candidate optical paths available between nodes $A$ and $C$ (step 3). Hereafter, the VNTM initiates the provisioning of a new lightpath between nodes $a$ and $c$ (step 4). Once the optical path is provisioned, the virtual link $A-C$ is reestablished. Therefore, by considering PCE, an MLR scheme can coordinate path computation while reducing signaling overhead and delay, hence avoiding the additional components and extended capabilities required at the NEs.

Despite the advantages provided by PCE schemes, enabling interaction between the NEs and a PCE might be very sophisticated (e.g., consider a fully-integrated MLR scheme), since the PCEP deployment into NEs is not native in current NEs.

In addition, in multiple failure scenarios, the coordination of recovery actions is required in order to avoid sending duplicated path computation queries to a PCE. Otherwise, some contention process must be done by a PCE. Therefore, the coordination of actions must be done either by the MLR or the PCE scheme.

On the other hand, SDN is gaining momentum in recent years as a centralized control architecture. The rationale behind SDNs is to enable the programmability of the forwarding table of NEs by decoupling the control plane and data planes. In a SDN CGN the mechanism associated to traffic forwarding (data plane) is located in the NEs, but control planes features are embedded in a separate hardware entity, called Remote Controller, which is typically a stand-alone server.

The flexibility provided by SDNs for real-time programming of the traffic flow has been well received in network research. This is because network vendors are unwilling to expose the internal operation of their network products mainly due to business policies. One of the possible solutions facilitating a real deployment of SDNs is OpenFlow. OpenFlow is a 
protocol used to program the forwarding table of a NE by means of a Remote Controller. With OpenFlow the traffic flow can be controlled using several parameters such as, VLAN ID, source/destination IP/MAC address, or a TCP port. It is worth mentioning that "flow" in the jargon of OpenFlow refers to packets or circuits (optical circuit); hence, optical circuit parameters can be also employed for controlling the direction of a flow, e.g., an optical wavelength.

OpenFlow can be considered as a useful tool with the aim of designing more advanced MLR schemes. The rationale for this assessment is that OpenFlow offers a more flexible interface to configure the forwarding plane of NEs from different vendors, compared to conventional management protocols such as, SNMP or NETCONF. The flexibility of OpenFlow substantially reduces the complexity of an MLR scheme. This reduction of complexity leads to agile MLR recovery schemes.

Figure 11 illustrates how a MLR scheme can leverage the features provided by OpenFlow. Let's assume a failure in the optical link $c-d$ pops up. Immediately, this failure affects the traffic forwarded by router $C$ to router $D$, what motivates that an IP-NMS triggers a message to the MLR scheme about the failure event (step 1). Then, the MLR scheme sends a query to the Remote Controller, requesting to modify the forwarding table of optical node $c$ (step 2). The Remote Controller does it, and hence sends an OpenFlow message to modify the forwarding table of optical node $c$, so that from that time on: i) the delay sensible traffic (Video traffic) destined to router $D$ is routed along the optical path $c-b-d$, and; ii) the non-sensible delay traffic (data traffic) destined to router $D$ is routed along the optical path $c-a-b-d$ (see step 3). This is so, due to the lack of network resources on the optical link $c-d$ to convey all traffic from router $C$ to router $D$.

Finally, we can conclude that MLR schemes along with OpenFlow can achieve fine-granularity related to the selection of recovery paths as proved by authors in [87], [88]. This is mainly rooted on the fact that by means of OpenFlow is possible to route traffic at different granularity levels, i.e., based on a TCP port, IP address or optical wavelength. It is no doubt that this routing flexibility enhances the efficiency of an MLR scheme.

Nevertheless, despite the strong research efforts devoted to SDN networks and PCE schemes, yet key issues must be addressed such as, the security mechanisms for both the remote controller and for the PCE in order to avoid malicious attacks [89], [90].

\section{OPEN ISSUES AND RESEARCH TRENDS}

In this section, we want to summarize and explicitly highlight the major research trends currently gathering and driving most efforts on the CGNs resilience management arena. Table III shows a collection of future research proposals related to the resilience arena.

\section{A. The Integration of Third Party Systems}

The integration of third party (centralized) systems consists in removing certain control features from the NEs such as, routing capabilities, and embed them into dedicated hardware

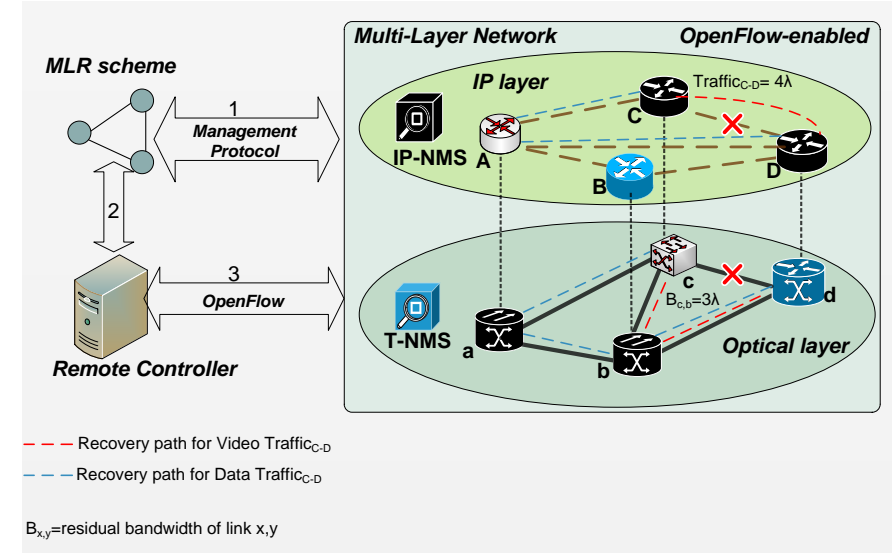

Figure 11. Integration of SDN in CGNs.

entities. A well-known outcome of this practice is the advent of third-party systems and new network architectures such as, the PCE or SDNs (being OpenFlow as the major enabler of a SDN), which adopts a centralized control approach. The adoption of centralized control architectures is driven by the ever-increasing deployment of new Internet services, demanding customized requirements from the control plane. This increase of signaling overhead is shifting the pendulum from distributed to centralized control schemes.

By means of both PCE and SDN is possible to enhance the operation of MLR schemes because recovery paths computations are done by an entity strictly devoted for this purpose. This operational enhancement opens the door to a diverse set of TE features that might be difficult to deploy in distributed control architectures mainly due to multi-vendor interoperability issues. It is worth mentioning that SDN and OpenFlow are not mutually exclusive, i.e., they can jointly coexist in a CGN scenario. The efficiency of this integration is proved by authors in [104], [105]. Authors propose the use of a PCE scheme for path computation purposes, whereas SDN is use for provisioning tasks. These studies demonstrate that the combine use of both PCE and SDN yields low complexity and low provisioning times.

Based on the strong research efforts devoted to both PCE and SDN, it is not doubt that these two schemes will continue gaining momentum in the resilience management arena.

\section{B. Hybrid NEs}

A different trend gaining momentum in nowadays network research focuses on combining the functionalities of both IP/MPLS and Optical NEs into a single hardware device referred to as Hybrid node. Several network vendors such as Juniper are offering hybrid nodes as part of their product catalog. This trend is represented in the Juniper PTX, a node with both routing and wavelength switching capabilities [24].

In hybrid network architectures, most of the correlationinduced problems and the interoperability issues described in this paper might be neglected, since there is no need for coordination amount NE with different technologies. However, the well-known intra-layer operability issues introduced by multi-vendor settings still need to be addressed. In addition, it 
Table III

REsEARCH TRENDS DEALING With THE MANAGEMENT OF RESILIENCE.

\begin{tabular}{|c|l|}
\hline SDN & [87], [91], [92], [93], [94] \\
\hline PCE & {$[95],[96],[97],[85],[98],[99]$} \\
\hline ILSA and Service-Oriented schemes & {$[100],[101],[42],[102],[103]$} \\
\hline
\end{tabular}

is worth mentioning that recovery schemes for hybrid network must be able to solve complex failure scenarios.

\section{Adoption of new Communication Models}

Finally, there is no doubt that the negative issues inherent to current routing and addressing schemes have a negative impact on the management of resilience. This is rooted in the fact that the host-oriented communication mode hinders the graceful deployment of Mobility and TE features. In addition, it is well known in the literature the issues related to the exhaustion of the available addressing space. This has lead to new -larger- addressing schemes -clean-slate- proposals suck as IPv6, capable of handling the demand of addresses. However, the migration from current IPv4 to IPv6 has been slow, mainly because of the interoperability issues.

On one hand, it is difficult to adopt current TE features aiming at increasing MLR schemes performance. Furthermore, the rapid change of addresses produced in mobile scenarios leads to suboptimal recovery path computations. In a similar manner, the interoperability issues in multi-addressing scenarios, also lead to suboptimal management of resilience, because the isolation imposed by two addressing schemes reduces the set of available recovery paths.

Fortunately, the negative effects of these issues may be reduced by means of new -non-disruptive- architectures, such as ILSAs. ILSAs schemes offer an alternative to the conventional host-oriented communication model, by adopting a service-oriented communication model. In a service-oriented communication model both routing and addressing features are not longer based on a particular destination address, rather multiple-destination addresses might be used according to the demanded service. This eases the deployment of Mobility and TE features, as well as it mitigates the interoperability issues in multi-addressing scenarios [106], [107], [108].

Nevertheless, without overlooking the strong research efforts devoted to ILSA schemes, there is scarce information available in the literature related to the study of ILSA schemes to enhance network resilience capabilities. It is reasonable to consider that since the deployment of ILSA schemes is becoming a widespread practice among network carriers, interaction between ILSA and MLR or PCE/SDN schemes might be required.

\section{CONCLuSions}

This paper provides a comprehensive survey of current Multi-Layer Resilience (MLR) schemes. To this end, we distil the critical weaknesses and limitations strongly preventing network operators to efficiently manage resilience in Carrier-Grade Networks (CGNs) based on the convergence of IP/MPLS and Optical technologies. We show that coordination among network layers (cross-layer coordination) is significantly important for MLR schemes. Indeed, crosslayer coordination is mainly affected by the interoperability of the management planes of Network Elements (NEs) with different technologies (IP or Optical) and network vendors. It is worth mentioning that notable advances have been made in the control plane concerning the cross-layer coordination in multi-layer CGNs, e.g., GMPLS, ASON, SDNs; however, few technological advances have been made related to the management plane.

\section{ACKNOWLEDGMENTS}

This work was supported by the Spanish Ministry of Economy under contract TEC2012-34682 (partially funded by FEDER), and the Catalan Research Council (CIRIT) under contract 2014SGR371.

\section{REFERENCES}

[1] T. Lehman, Xi Yang, N. Ghani, Feng Gu, Chin Guok, I. Monga, and B. Tierney. Multilayer networks: an architecture framework. Communications Magazine, IEEE, 49(5):122 -130, may 2011.

[2] J.E. Rathke. Surveys of emerging control plane initiatives. In Optical Fiber Communication Conference and Exposition and the National Fiber Optic Engineers Conference (OFC/NFOEC), 2013, pages 1-7, March 2013.

[3] V.W.S. Chan, J. Jacobs, I. Jacobs, and Zhang Lei. Scalable control plane architecture for optical flow switched networks. In Optical Fiber Communication Conference and Exposition (OFC/NFOEC), 2011 and the National Fiber Optic Engineers Conference, pages 1-3, March 2011.

[4] IETF. Generalized Multi-Protocol Label Switching (GMPLS) Architecture (RFC 3945), October 2004.

[5] Open Networking foundation. Openflow Switch Specification Version 1.1.0 http://www.openflow.org/documents/openflow-spec-v1.1.0.pdf.

[6] ITU-T. Architecture for the Automatically Switched Optical Network (ASON) - Rec. 8080/Y.1304, 2001.

[7] R. Enns, M. Bjorklund, J. Schoenwaelder, and Bierman A. Network Configuration Protocol (NETCONF), RFC-6241, June 2011.

[8] J.P Vasseur et al. Network Recovery: Protection and Restoration of Optical, SONET-SDH, IP, and MPLS. David Clark, 2004.

[9] P. Cholda and A. Jajszczyk. Recovery and Its Quality in Multilayer Networks. Lightwave Technology, Journal of, 28(4):372-389, Feb 2010.

[10] N. Ghani, Qing Liu, D. Benhaddou, N.S.V. Rao, and T. Lehman. Control plane design in multidomain/multilayer optical networks. Communications Magazine, IEEE, 46(6):78-87, June 2008.

[11] T. Morioka, Y. Awaji, R. Ryf, P. Winzer, D. Richardson, and F. Poletti. Enhancing optical communications with brand new fibers. Communications Magazine, IEEE, 50(2):s31-s42, 2012.

[12] Dayou Qian, Ming-Fang Huang, E. Ip, Yue-Kai Huang, Yin Shao, Junqiang Hu, and Ting Wang. High Capacity/Spectral Efficiency 101.7Tb/s WDM Transmission Using PDM-128QAM-OFDM Over 165-km SSMF Within C- and L-Bands. Lightwave Technology, Journal of, 30(10):1540-1548, 2012.

[13] http://metroethernetforum.org/.

[14] IEEE Standard for Local and Metropolitan Area Networks - Virtual Bridged Local Area Networks, Amendment 4: Provider Bridges. IEEE Std 802.1ad-2005 (Amendment to IEEE Std 8021Q-2005), pages 1 -60, 2006. 
[15] IEEE Standard for Local and metropolitan area networks - Virtual Bridged Local Area Networks Amendment 7: Provider Backbone Bridges. IEEE Std 802.1ah-2008 (Amendment to IEEE Std 802.1Q2005), pages $1-110,142008$.

[16] IEEE Draft Standard for Local and Metropolitan Area NetworksVirtual Bridged Local Area Networks Amendment: Provider Backbone Bridge Traffic Engineering (PBB-TE) Infrastructure Segment Protection. IEEE P802.1Qbf/D1.3, February 2011, pages 1-84, 2011.

[17] IEEE Standard for Local and Metropolitan Area Networks - Virtual Bridged Local Area Networks Amendment 5: Connectivity Fault Management. IEEE Std 802.1ag - 2007 (Amendment to IEEE Std 802.1Q - 2005 as amended by IEEE Std 802.1ad - 2005 and IEEE Std 802.1ak - 2007), pages $1-260,2007$.

[18] O. Gerstel, M. Jinno, A. Lord, and S.J.B. Yoo. Elastic optical networking: a new dawn for the optical layer? Communications Magazine, IEEE, 50(2):s12-s20, February 2012.

[19] W. Ramirez, X. Masip-Bruin, M. Yannuzzi, D. Montero, A. Martinez, and V. Lopez. Network coding-based protection scheme for Elastic Optical Networks. In Design of Reliable Communication Networks (DRCN), 2014 10th International Conference on the, pages 1-8, April 2014.

[20] Bowen Chen, Jie Zhang, Yongli Zhao, Chunhui Lv, Wei Zhang, Shanguo Huang, Xian Zhang, and Wanyi Gu. Multi-link failure restoration with dynamic load balancing in spectrum-elastic optical path networks. Optical Fiber Technology, 18(1):21 - 28, 2012.

[21] Y. Sone, A. Watanabe, W. Imajuku, Y. Tsukishima, B. Kozicki, H. Takara, and M. Jinno. Bandwidth Squeezed Restoration in Spectrum-Sliced Elastic Optical Path Networks (SLICE). Optical Communications and Networking, IEEE/OSA Journal of, 3(3):223-233, March 2011.

[22] M. Bocci et al. A Framework for MPLS in TransportNetworks, IETF RFC 5921, July 2010.

[23] R. Cafini, W. Cerroni, C. Raffaelli, and M. Savi. Standard-based approach to programmable hybrid networks. Communications Magazine, IEEE, 49(5):148 -155, may 2011.

[24] PTX Series Packet Transport Routers, http://www.juniper.net/us/en/local/pdf/datasheets/1000364-en.pdf.

[25] W. Ramirez, X. Masip-Bruin, M. Yannuzzi, R. Serral-Gracia, A. Martinez, and M. S. Siddiqui. A Survey and Taxonomy of ID/Locator Split Architectures. Comput. Netw., 60:13-33, February 2014.

[26] B. Ahlgren, C. Dannewitz, C. Imbrenda, D. Kutscher, and B. Ohlman A survey of information-centric networking. Communications Magazine, IEEE, 50(7):26-36, July 2012.

[27] W. Lai and D. McDysan. Network Hierarchy and Multilayer Survivability, November 2002.

[28] R. Duque, V. Lopez, A. Gonzalez, O.G. de Dios, and J.P. FernandezPalacios. Cost evaluation of the integration of IP/MPLS and WDM elements. In Optical Fiber Communication Conference and Exposition and the National Fiber Optic Engineers Conference (OFC/NFOEC), 2013, pages 1-3, 2013.

[29] International Telecommunications Union (ITU). Amplified multichannel dense wavelength division multiplexing applications with single channel optical interfaces (ITU-T Recommendation G.698.2), November 2009.

[30] D. Papadimitriou. E. Mannie. Recovery (Protection and Restoration) Terminology for Generalized Multi-Protocol Label Switching (GMPLS)., March 2006.

[31] W. Ramirez, X Masip-Bruin, M. Yannuzzi, E. Marin-Tordera, A. Martinez, and V. Lopez. A Techno-Economic Study of Network Coding Protection Schemes. Proceedings of IEEE GLOBECOM 2014 ., 2014.

[32] A. Fumagalli and L. Valcarenghi. IP restoration vs. WDM protection: is there an optimal choice? Network, IEEE, 14(6):34-41, nov/dec 2000.

[33] F. Munoz, V. Lopez, O.G. de Dios, and J.P. Fernandez-Palacios. Multilayer restoration in hierarchical IP/MPLS over WSON networks. In Networks and Optical Communications (NOC), 2012 17th European Conference on, pages 1-6, 2012.

[34] Y. Sone, Wataru Imajuku, and M. Jinno. Multiple Failure Recovery of Optical Paths Using GMPLS Based Restoration Scheme Escalation. In Optical Fiber Communication and the National Fiber Optic Engineers Conference, 2007. OFC/NFOEC 2007. Conference on, pages 1-3, 2007.

[35] Ran Li, Xiaoliang Wang, and Xiaohong Jiang. Network survivability against region failure. In Signal Processing, Communications and Computing (ICSPCC), 2011 IEEE International Conference on, pages $1-6,2011$
[36] E. Mannie D. Papadimitriou. RFC-4428, Analysis of Generalized Multi-Protocol Label Switching (GMPLS)-based Recovery Mechanisms (including Protection and Restoration).

[37] Wensheng He and AK. Somani. Comparison of Protection Mechanisms: Capacity Efficiency and Recovery Time. In Communications, 2007. ICC '07. IEEE International Conference on, pages 2218-2223, June 2007.

[38] A. Haider and R. Harris. Recovery techniques in next generation networks. Communications Surveys Tutorials, IEEE, 9(3):2 -17, quarter 2007.

[39] O. Gerstel and R. Ramaswami. Optical layer survivability: a postbubble perspective. Communications Magazine, IEEE, 41(9):51 - 53, sept. 2003

[40] A. Zinin A. Atlas. Basic Specification for IP Fast Reroute: Loop-Free Alternates, 2008.

[41] G. Enyedi A. Csaszar J. Tantsura M. Konstantynowicz R. White M. Shand A. Atlas, R. Kebler. An Architecture for IP/LDP FastReroute Using Maximally Redundant Trees, 2013.

[42] P. Babarczi, J. Tapolcai, A. Pasic, S.R. Darehchi, and Pin-Han Ho. New addressing scheme to increase reliability in MPLS with network coding. In Design of Reliable Communication Networks (DRCN), 2013 9th International Conference on the, pages 36-43, 2013.

[43] R. Martinez, A. Castro, R. Casellas, R. Munoz, L. Velasco, R. Vilalta, and J. Comellas. Experimental validation of dynamic restoration in GMPLS-controlled multi-layer networks using PCE-based global concurrent optimization. In Optical Fiber Communication Conference and Exposition and the National Fiber Optic Engineers Conference (OFC/NFOEC), 2013, pages 1-3, March 2013.

[44] Huan Chen and Po-Kai Tseng. A low complexity shortest path tree restoration scheme for IP networks. Communications Letters, IEEE, 14(6):566-568, June 2010.

[45] Bingli Guo, Shanguo Huang, Pei Luo, HaoTian Huang, Jie Zhang, and Wanyi Gu. Dynamic Survivable Mapping in IP Over WDM Network. Lightwave Technology, Journal of, 29(9):1274-1284, May 2011.

[46] Muriel Medard, Richard A. Barry, Steven G. Finn, Wenbo He, and Steven S. Lumetta. Generalized loop-back recovery in optical mesh networks. IEEE/ACM Trans. Netw., 10(1):153-164, February 2002.

[47] S. Azodolmolky, M. Klinkowski, Y. Pointurier, M. Angelou, D. Careglio, J. Sole-Pareta, and I. Tomkos. A Novel Offline Physical Layer Impairments Aware RWA Algorithm With Dedicated Path Protection Consideration. Lightwave Technology, Journal of, 28(20):30293040 , Oct 2010

[48] Jitender S. Deogun L. Kong, M. Ali. Building Redundant Multicast Trees for Preplanned Recovery in WDM optical networks. High Speed Networks, 15:379-398, 2006.

[49] Menglin Liu, M. Tornatore, and B. Mukherjee. Efficient shared subconnection protection in mixed-line-rate optical WDM networks. Optical Communications and Networking, IEEE/OSA Journal of, 5(11):12271235, Nov 2013.

[50] R. Yadav, R.S. Yadav, and H. Singh. Enhanced Intercycle Switching in p-Cycle Survivability for WDM Networks. Optical Communications and Networking, IEEE/OSA Journal of, 2(11):961-966, November 2010.

[51] H. Overby, G. Biczok, P. Babarczi, and J. Tapolcai. Cost comparison of $1+1$ path protection schemes: A case for coding. In Communications (ICC), 2012 IEEE International Conference on, pages 3067-3072.

[52] A. Giorgetti, L. Valcarenghi, F. Cugini, and P. Castoldi. PCE-Based Dynamic Restoration in Wavelength Switched Optical Networks. In Communications (ICC), 2010 IEEE International Conference on, pages $1-6$, may 2010.

[53] B.T. Mangara and F.W. Leuschner. Automated dynamic protection planning for improved survivability of DWDM optical networks: performance analysis for distributed restoration algorithms. In AFRICON, 2004. 7th AFRICON Conference in Africa, volume 2, pages 977 - 979 Vol.2, sept. 2004.

[54] J. Perello, S. Spadaro, F. Agraz, M. Angelou, S. Azodolmolky, Y. Qin, R. Nejabati, D. Simeonidou, P. Kokkinos, E. Varvarigos, S. Al Zahr, M. Gagnaire, and I. Tomkos. Experimental evaluation of centralized failure restoration in a dynamic impairment-aware all-optical network. In Optical Fiber Communication Conference and Exposition (OFC/NFOEC), 2011 and the National Fiber Optic Engineers Conference, pages $1-3$, march 2011.

[55] G. Mohan, C. Siva Ram Murthy, and A.K. Somani. Efficient algorithms for routing dependable connections in WDM optical networks. Networking, IEEE/ACM Transactions on, 9(5):553 -566, oct 2001. 
[56] E. Bouillet and J.-F. Labourdette. Distributed computation of shared backup path in mesh optical networks using probabilistic methods. Networking, IEEE/ACM Transactions on, 12(5):920 - 930, oct. 2004.

[57] Yang Qin, L. Mason, and Ke Jia. Study on a joint multiple layer restoration scheme for IP over WDM networks. Network, IEEE, 17(2):43 - 48, mar/apr 2003.

[58] C. Pinart. A multilayer fault localization framework for IP over alloptical multilayer networks. Network, IEEE, 23(3):4 -9, may-june 2009.

[59] Lei Lei, Aibo Liu, and Yuefeng Ji. A joint resilience scheme with interlayer backup resource sharing in IP over WDM networks. Communications Magazine, IEEE, 42(1):78 - 84, jan 2004.

[60] P. Pacharintanakul. The effects of multi-layer traffic on the survivability of IP-over-WDM networks. In Proceedings of the 2009 IEEE international conference on Communications, ICC'09, pages 2354-2359, Piscataway, NJ, USA, 2009. IEEE Press.

[61] E. Palkopoulou, D.A. Schupke, and T. Bauschert. Shared backup router resources: realizing virtualized network resilience. Communications Magazine, IEEE, 49(5):140 -146, may 2011.

[62] K. Kompella, Y. Rekhter, A. Banerjee, J. Drake, G. Bernstein, D. Fedyk, E. Mannie, D. Saha, and V. Sharma. OSPF Extensions in Support of Generalized MPLS, draft-kompella-ospf-gmpls-extensions02.txt, January 2002.

[63] K. Sato, N. Yamanaka, Y. Takigawa, M. Koga, S. Okamoto, K. Shiomoto, E. Oki, and W. Imajuku. GMPLS-based photonic multilayer router (Hikari router) architecture: an overview of traffic engineering and signaling technology. Communications Magazine, IEEE, 40(3):96 -101 , mar 2002.

[64] M. Yannuzzi, X. Masip-Bruin, O.G. de Dios, C.G. Argos, M. Maciejewski, and J. Altmann. Bridging the interoperability gap between the Internet and optical network management systems. In Networks and Optical Communications (NOC), 2011 16th European Conference on, pages $177-180$, july 2011.

[65] L. Yang, R. Dantu, T. Anderson, and R. Gopal. Forwarding and Control Element Separation (ForCES) Framework, RFC 3746, Apr 2004.

[66] Qiong Zhang, A.L. Chiu, Xi Wang, P. Palacharla, and M. Sekiya. Restoration design with selective optical bypass in IP-over-optical networks. In Optical Fiber Communication Conference and Exposition (OFC/NFOEC), 2012 and the National Fiber Optic Engineers Conference, pages 1-3, March 2012.

[67] Jijun Zhao, Sujian Wang, and Zhiyuan Tang. Integrated Multi-layer Network Survivability Based on GMPLS to Improve Fault Recovery Time. In Computer Engineering and Technology, 2009. ICCET '09. International Conference on, volume 2, pages 126-130, Jan 2009.

[68] B. Nleya and E. Nyakwende. Survivability: Wavelength Recovery for Node and Link Failure in All Optical Networks. In Broadband Communications, Information Technology Biomedical Applications, 2008 Third International Conference on, pages 492-498, Nov 2008.

[69] V. Lopez, J.A. Hernandez, O. Gonzalez de Dios, J.F. Palacios, and J. Aracil. Multilayer Traffic Engineering for IP Over WDM Networks Based on Bayesian Decision Theory. Optical Communications and Networking, IEEE/OSA Journal of, 2(8):515-529, August 2010.

[70] S. Martinez, V. Lopez, M. Chamania, O. Gonzalez, A. Jukan, and J.P. Fernandez-Palacios. Assessing the performance of multi-layer path computation algorithms for different PCE architectures. In Optical Fiber Communication Conference and Exposition and the National Fiber Optic Engineers Conference (OFC/NFOEC), 2013, pages 1-3, March 2013.

[71] B. de la Cruz, O. Gonzalez de Dios, V. Lopez, and J.P. FernandezPalacios. Operational expenditures savings in IP/MPLS over DWDM networks by Multi-layer restoration. In Optical Fiber Communications Conference and Exhibition (OFC), 2014, pages 1-3, March 2014.

[72] A.L. Chiu, G. Choudhury, M.D. Feuer, J.L. Strand, and S.L. Woodward. Integrated Restoration for Next-Generation IP-Over-Optical Networks. Lightwave Technology, Journal of, 29(6):916 -924, march15, 2011.

[73] J. Moy. OSPF Version 2, April 1998.

[74] M. Bjorklund. YANG - A Data Modeling Language for the Network Configuration Protocol (NETCONF), October 2010.

[75] Jijun Zhao, Lei Lei, Yuefeng Ji, and Daxiong Xu. Integrated multilayer survivability strategy with inter-layer signaling. In Communication Technology Proceedings, 2003. ICCT 2003. International Conference on, volume 1, pages $612-616$ vol.1, april 2003.

[76] D. Coudert, S. Perennes, H. Rivano, and Marie-Emilie Voge. Shared Risk Resource Groups and Survivability in Multilayer Networks. In Transparent Optical Networks, 2006 International Conference on, volume 3, pages $235-238$, june 2006.
[77] G. Booker, A. Sprintson, E. Zechman, C. Singh, and S. Guikema. Efficient traffic loss evaluation for transport backbone networks. Comput. Netw., 54(10):1683-1691, July 2010.

[78] J. Lang. Link Management Protocol (LMP), RFC-4204, 2005.

[79] M. Chamania, E. Demirbilek, A. Jukan, X. Masip-Bruin, and M. Yannuzzi. Using BPEL workflow processing for cross-layer orchestrations in IP-over-optical networks: A proof of concept. In Network Operations and Management Symposium (NOMS), 2012 IEEE, pages 1191-1194, April 2012.

[80] Choonho Son, Junsuk Oh, Kyoung-Ho Lee, Kieung Kim, and Jaehyung Yoo. Efficient physical topology discovery for large OSPF networks. In Network Operations and Management Symposium, 2008. NOMS 2008. IEEE, pages 325-330, 2008.

[81] A. Farrel and J.-P Vasseur. A Path Computation Element (PCE)-Based Architecture, RFC 4655, August 2006.

[82] G. Lee, Y. Bernstein and W. Imajuku. Framework for GMPLS and Path Computation Element (PCE) Control of Wavelength Switched Optical Networks (WSONs), RFC 6163, April 2011.

[83] JL. Le Roux JP. Vasseur. Path Computation Element (PCE) Communication Protocol (PCEP), March 2009.

[84] D.A. Schupke and F. Rambach. Path computation element; An enabler for automated network operation. In Networks and Optical Communications (NOC), 2011 16th European Conference on, pages $12-15$, july 2011.

[85] M. Chamania, O.G. de Dios, V. Lopez, M. Cuaresma, M. Drogon, A. Jukan, X. Masip-Bruirr, and M. Yannuzzi. Coordinated computation of multi-layer paths via inter-layer PCE communication: Standards, interoperability and deployment. In Communications (ICC), 2012 IEEE International Conference on, pages 6926-6931, 2012.

[86] N. Andriolli, F. Cugini, L. Valcarenghi, P. Castoldi, and A. Welin. Virtual network topology manager (VNTM) and path computation element (PCE) cooperation in multi-layer GMPLS networks. In Optical Fiber Communication - incudes post deadline papers, 2009. OFC 2009. Conference on, pages 1-3, March 2009.

[87] Lei Liu, Dongxu Zhang, T. Tsuritani, R. Vilalta, R. Casellas, Linfeng Hong, I. Morita, Hongxiang Guo, Jian Wu, R. Martinez, and R. Munoz. Field Trial of an OpenFlow-Based Unified Control Plane for Multilayer Multigranularity Optical Switching Networks. Lightwave Technology, Journal of, 31(4):506-514, Feb 2013.

[88] H. Kudou, M. Shimamura, T. Ikenaga, and M. Tsuru. Effects of routing granularity on communication performance in OpenFlow networks. In Communications, Computers and Signal Processing (PacRim), 2011 IEEE Pacific Rim Conference on, pages 590-595, Aug 2011.

[89] R. Kloti, V. Kotronis, and P. Smith. OpenFlow: A security analysis. In Network Protocols (ICNP), 2013 21st IEEE International Conference on, pages 1-6, Oct 2013.

[90] S. Son, Seungwon Shin, V. Yegneswaran, P. Porras, and Guofei Gu. Model checking invariant security properties in OpenFlow. In Communications (ICC), 2013 IEEE International Conference on, pages 1974-1979, June 2013.

[91] A. Giorgetti, F. Paolucci, F. Cugini, and P. Castoldi. Fast restoration in SDN-based flexible optical networks. In Optical Fiber Communications Conference and Exhibition (OFC), 2014, pages 1-3, March 2014.

[92] R. Alvizu and G. Maier. Can open flow make transport networks smarter and dynamic? An overview on transport SDN. In Smart Communications in Network Technologies (SaCoNeT), 2014 International Conference on, pages 1-6, June 2014.

[93] Frank Slyne, Nattapong Kituswan, Seamas McGettrick, David B. Payne, and Marco Ruffini. Design and experimental test of 1:1 endto-end protection for LR-PON using an SDN multi-tier control plane. In Optical Communication (ECOC), 2014 European Conference on, pages 1-3, Sept 2014.

[94] O. Gonzalez de Dios, V. Loopez, C. Haya, C. Liou, Ping Pan, G. Grammel, J. Antich, and J.P. Fernandez-Palacios. Traffic engineering database dissemination for multi-layer SDN orchestration. In Optical Communication (ECOC 2013), 39th European Conference and Exhibition on, pages 1-3, Sept 2013.

[95] J. Ahmed, C. Cavdar, P. Monti, and L. Wosinska. Survivability strategies for PCE-based WDM networks offering high reliability performance. In Optical Fiber Communication Conference and Exposition and the National Fiber Optic Engineers Conference (OFC/NFOEC), 2013, pages 1-3, March 2013.

[96] A. Castro, L. Velasco, J. Comellas, and G. Junyent. Dynamic restoration in multi-layer IP/MPLS-over-flexgrid networks. In Design of Reliable Communication Networks (DRCN), 2013 9th International Conference on the, pages 155-162, March 2013. 
[97] P. Iovanna, A. Germoni, F. Testa, G. Cossu, V. Lopez, and R. Sabella. Multilayer control for packet-optical networks [invited]. Optical Communications and Networking, IEEE/OSA Journal of, 5(10):A86A99, Oct 2013.

[98] J.S. Dantas, D. Careglio, R.M. Silveira, W.V. Ruggiero, and J. SolePareta. PCE algorithm for traffic grooming and QoS in multilayer/multi-domain IP over WDM networks. In Transparent Optical Networks (ICTON), 2011 13th International Conference on, pages 1-5, June 2011.

[99] Yongli Zhao, Jie Zhang, Min Zhang, Yuefeng Ji, and Wanyi Gu. DREAM: dual routing engine architecture in multilayer and multidomain optical networks. Communications Magazine, IEEE, 51(5):118127, May 2013.

[100] F. Coras, D. Saucez, L. Jakab, A. Cabellos-Aparicio, and J. DomingoPascual. Implementing a BGP-free ISP core with LISP. In Global Communications Conference (GLOBECOM), 2012 IEEE, pages 2772 2778, Dec 2012.

[101] H. Urabayashi, M. Yamamoto, and T. Yagyu. Performance Evaluation of Robustness of Inter-Layer 3 Networking with ID/Locator Separation Architecture. In World Telecommunications Congress (WTC), 2012, pages 1-6, March 2012.

[102] C.E. Abosi, R. Nejabati, and D. Simeonidou. A Novel Service Composition Mechanism for the Future Optical Internet. Optical Communications and Networking, IEEE/OSA Journal of, 1(2):A106A120, July 2009.

[103] G. Zervas, V. Martini, Y. Qin, E. Escalona, R. Nejabati, D. Simeonidou, F. Baroncelli, B. Martini, K. Torkmen, and Piero Castoldi. ServiceOriented Multigranular Optical Network Architecture for Clouds. Optical Communications and Networking, IEEE/OSA Journal of, 2(10):883-891, October 2010.

[104] A. Mayoral, R. Vilalta, R. Munoz, R. Casellas, and R. Martinez. Experimental validation of automatic lightpath establishment integrating OpenDayLight SDN controller and Active Stateful PCE within the ADRENALINE testbed. In Transparent Optical Networks (ICTON), 2014 16th International Conference on, pages 1-4, July 2014.

[105] G.C. Santos, A.T. Hirata, M.B. Trindade, J.R.F. Oliveira, F.N.C Van't Hooft, J.C.R.F. Oliveira, M. Siqueira, and C. Esteve Rothenberg. Employment of IA-RWA in Virtual Optical Networks Using a PCE Implemented as a SDN Application. In Computer Networks and Distributed Systems (SBRC), 2014 Brazilian Symposium on, pages $207-$ 213, May 2014.

[106] X. Misseri, J. Rougier, and D. Saucez. Internet routing diversity for stub networks with a Map-and-Encap scheme. In Communications (ICC), 2012 IEEE International Conference on, pages 2861-2866, June 2012.

[107] M. Al-Naday, M. Reed, D. Trossen, and Kun Yang. Information resilience: source recovery in an information-centric network. Network, IEEE, 28(3):36-42, May 2014.

[108] F. Francois, Ning Wang, K. Moessner, S. Georgoulas, and R. de Oliveira Schmidt. Leveraging MPLS Backup Paths for Distributed Energy-Aware Traffic Engineering. Network and Service Management, IEEE Transactions on, 11(2):235-249, June 2014. 